\title{
Higgs-bosons couplings to quarks and leptons in the supersymmetric Standard Model with a gauge singlet
}

\author{
Andreas Crivellin $^{a}$ and Youichi Yamada ${ }^{b}$ \\ a Theory Division, CERN, \\ CH-1211 Geneva 23, Switzerland \\ ${ }^{b}$ Department of Physics, Tohoku University, \\ Sendai 980-8578, Japan \\ E-mail: andreas.crivellin@cern.ch, yamada@tuhep.phys.tohoku.ac.jp
}

\begin{abstract}
The loop corrections to the couplings of Higgs bosons to quarks and charged leptons are calculated within supersymmetric versions of the Standard Model, extended by a gauge singlet. The effective couplings of the $\mathrm{SU}(2)_{L}$ doublet and singlet Higgs bosons to quarks and leptons, induced by sfermion loops, are derived. Analytic expressions for the case of generic sfermion flavour mixing, including the complete resummation of all chirally-enhanced contributions are presented. These results are important in scenarios in which the mixing between singlet and doublet components of Higgs bosons is small, and the (pseudo) scalar component of the doublet is light. The calculated loop effects can have important consequences in flavour physics, especially for $\Delta F=2$ processes.
\end{abstract}

Keywords: Supersymmetry Phenomenology

ARXiv EPrint: 1508.02855 


\section{Contents}

1 Introduction 1

2 SUSY models with a gauge singlet $\quad 3$

2.1 Mixing of Higgs bosons $\quad 6$

2.2 Flavour parameters/violations 8

3 Quark and lepton self-energies $\quad 9$

4 Renormalization and threshold corrections 13

5 Effective Higgs couplings to quarks and leptons $\quad \mathbf{1 5}$

$\begin{array}{lll}5.1 \text { Couplings to doublet Higgs bosons } & 15\end{array}$

$\begin{array}{ll}5.2 \text { Effective singlet-fermion couplings } & 16\end{array}$

$\begin{array}{ll}\text { 5.2.1 Gluino and bino induced couplings } & 16\end{array}$

$\begin{array}{ll}\text { 5.2.2 Chargino and neutralino induced couplings } & 17\end{array}$

$\begin{array}{lll}5.3 & \text { Higgs couplings in the physical basis } & 18\end{array}$

6 Numerical results $\quad 20$

6.1 Flavour-conserving couplings 21

$\begin{array}{ll}6.2 \text { Flavour-changing couplings } & 22\end{array}$

$\begin{array}{lll}6.2 .1 & \text { Leptons } & 22\end{array}$

6.2.2 Quarks 22

6.3 Effects in flavour-changing processes 23

$\begin{array}{llr}7 & \text { Conclusion } & 25\end{array}$

\section{Introduction}

The supersymmetric model with a gauge singlet scalar in addition to the usual particle content of the Minimal Supersymmetric Standard Model (MSSM) is a well motivated model, and was already proposed in the early days [1-3] of studies of Supersymmetry (SUSY), as a solution to the $\mu$ problem [4]. It receives even more attention today in light of the recent Higgs boson discovery $[5,6]$.

These models were for sometime even the focus of Higgs-boson studies in SUSY before the Higgs boson was actually discovered with the relatively low value of mass of $\sim 126 \mathrm{GeV}$, which can be accounted for in the MSSM. The reason was the fact, noticed already quite early in refs. [7-10], that the singlet coupling to the Higgs doublets allows to break the link of the Higgs quartic couplings to gauge couplings, typical of the MSSM. Supersymmetric models with additional gauge singlets can therefore accommodate for a value of the SM-like 
Higgs mass much larger than that allowed in the MSSM, at least for small values of $\tan \beta$. Before the Higgs boson discovery, this fact reconciled SUSY aficionados with the possibility that the LHC would find a Higgs boson far heavier than that predicted by the MSSM (see for example ref. [11]). It also possess the capability of reducing the amount of parameter fine tuning needed in order to obtain the correct Higgs mass (see for example [12-14]). Since then, it has become clear that a larger Higgs mass than that allowed in the MSSM is also possible for moderate to large large values of $\tan \beta[15,16]$ due to the possible doublet-singlet mixing contributions to the physical Higgs masses.

In addition to the original scale-invariant model, called the Next-to-Minimal Supersymmetric Standard Model (NMSSM), there exists other forms of the MSSM extended with a gauge singlet superfield. They differ by the singlet self interactions terms present in the superpotential. There is in particular the "Minimal Non-minimal Supersymmetric Standard Model" (MNSSM) [17-19] or nMSSM, and the PQ-NMSSM [4, 20-22]. In all these cases, the only other particles to which the singlet superfield $S$ couple are the Higgs doublet superfields $H_{u}$ and $H_{d}$. The models differ only in the way the Peccei-Quinn symmetry $\mathrm{U}(1)_{\mathrm{PQ}}[1,20,23]$ of the superpotential is explicitly broken at the electroweak scale, i.e. either through a cubic term in $S$, or a linear one. The phenomenological studies of both models amounts to a sizable part of all beyond-SM analyses [24-29].

Over the years, particular attention has been paid to the fact that the lightest pseudoscalar particle in these models, $a_{1}$, can be quite light (see for example refs. [30-33], and references therein). Apart from small admixtures with the CP-odd neutral scalar components of $H_{u}$ and $H_{d}$, the mass eigenstate $a_{1}$ is mainly the pseudoscalar component of the singlet superfield $S$. The lightest CP-even neutral Higgs boson, $h_{1}$, may be equally light, depending on the values of various parameters of the model. Potentially enormous consequence arise from this fact for Higgs studies at the LHC [31-34].

The presence of light particles in the spectrum clearly affects also the physics of flavoured mesons, modifying therefore the MSSM searches at the high-luminosity frontier. Indeed, the impact of very light Higgs bosons in $K$ - and $B$-meson decays, has been the subject of intensive studies (see e.g. refs. [25, 26, 35-37]). Flavour physics will keep playing an important role in the exclusion or detection of such light states through searches at the Belle II factory at SuperKEKB and with the LHCb program at CERN, in particular through the measurements of $B_{d}^{0}-\bar{B}_{d}^{0}$ and $B_{s}^{0}-\bar{B}_{s}^{0}$ mixing, and of the decay $B_{s} \rightarrow \mu^{+} \mu^{-}$. Direct searches for $a_{1}$ at the LEP [38, 39], $B$ factories [40-43], and the LHC [44, 45] have already reduced the parameter space of these models. Needless to say, direct searches of additional Higgs states will be able to probe whether singlet states are part of the Higgs sector or not.

It is therefore very important to know with a good precision the couplings of the Higgs bosons to quarks and leptons (of relevance for direct Higgs boson searches, flavour physics and also dark matter direct detection). Note that all Higgs mass eigenstates in this class of models, also those which are mainly singlet states, can couple to matter through their mixing with doublet Higgs states. Moreover, couplings of the singlet $S$ to matter fermion, even though they are vanishing at tree-level, are generated at the one-loop level as was first pointed out in refs. [46-48], and more recently in ref. [37]. However, these effective 
singlet-fermion couplings have not attracted much attention. Although some existing codes for these models (such as NMSSMCALC [49] and SPheno $[50,51]$ ) include these couplings partially, ${ }^{1}$ no complete formula including resummation effects are implemented. It is true that the experimentally found value of the Higgs mass puts some emphasis on low values of $\tan \beta$, while these loop effects are maximized for the largest possible values of $\tan \beta$. Nevertheless, these effects cannot be neglected. They should be taken into account until the final embedding of the MSSM with a singlet is -hopefully- experimentally discovered. See, for example, ref. [53] for the study of NMSSM with large $\tan \beta$.

In this paper we plan to revisit the effective couplings of the singlet Higgs to leptons and quarks, induced by SUSY particle loops. ${ }^{2}$ To this end we work in the approximation of large $\tan \beta$ where the loop effects are phenomenologically relevant. This implies that a certain amount of fine tuning is needed to reproduce the measured value of the Higgs mass, like it is the case in the MSSM as well. With respect to the calculations in refs. [46], we drop the assumption of minimal flavour violation but rather include the effects of flavourchanging soft parameters. We also analytically re-sum all chirally-enhanced effects (as done in ref. [54] for the MSSM) for threshold corrections to Yukawa couplings and to the CKM matrix. While also ref. [48] worked in the MSSM with generic flavour structure, ${ }^{3}$ we include the wino and bino contributions which are not included in ref. [48]. The results shown in this article are valid for all extension of the MSSM with a singlet, where the $\mu$ term is generated by the vacuum expectation value (VEV) of $S$, irrespectively of the singlet self-interaction terms allowed in the superpotential. ${ }^{4}$

This article is organized as follows: in section 2, we review the basic properties of SUSY standard models with an additional gauge singlet supermultiplet. In section 3 , the self energies of quarks and leptons generated by SUSY loops are calculated. Section 4 deals with the effective couplings of the singlet Higgs which are expressed in terms of these self energy. Section 5 shows our numerical results and finally we conclude in section 6 .

\section{SUSY models with a gauge singlet}

In this section we review the basics of models obtained adding a singlet field to the MSSM particle content. In these models the superpotential is given by:

$$
W=W_{\text {Yuk }}^{\mathrm{MSSM}}+\lambda S H_{u} \cdot H_{d}+f(S),
$$

where $W_{\text {Yuk }}^{\mathrm{MSSM}}$ contains the usual Yukawa structure:

$$
W_{\text {Yuk }}^{\mathrm{MSSM}}=U^{c} Y_{U} Q \cdot H_{u}-D^{c} Y_{D} Q \cdot H_{d}-E^{c} Y_{L} L \cdot H_{d}
$$

\footnotetext{
${ }^{1}$ In NMSSMTools [52] only the threshold corrections to the doublet Higgs Yukawa couplings but not the loop corrections to the singlet-quark couplings are implemented.

${ }^{2}$ Note that the charged Higgs boson loops also give the $\tan \beta$ enhanced contribution in the NMSSM [37]. We do not consider this contribution here, since it depends on the details of the Higgs potential.

${ }^{3}$ Ref. [48] has presented numerical results only for the minimal flavour violation case. Note also that the calculation in ref. [37] has included the $\tilde{t}_{L}-\tilde{c}_{L}$ mixing.

${ }^{4}$ We assume a a CP-conserving Higgs potential. A recent study of the $\mathrm{CP}$ violation in the Higgs sector in NMSSM is seen in refs. [55, 56].
} 
with $Q \equiv(U, D)^{T}$ and $L \equiv(N, E)^{T}$, the quark and lepton $\mathrm{SU}(2)_{L}$ doublet superfields; $U^{c}, D^{c}$, and $E^{c}$, the $\mathrm{SU}(2)_{L}$ singlet ones, and symbolizing the antisymmetric $\mathrm{SU}(2)$ invariant product. The part of the superpotential denoted by $f(S)$ contains the singlet self interactions that identify the specific model in this class. Before proceeding, we give also the soft SUSY-breaking scalar terms needed to specify these models:

$$
V_{\mathrm{soft}}=V_{\mathrm{soft}-\mathrm{Yuk}}^{\mathrm{MSSM}}+V_{\mathrm{soft}}^{\mathrm{bilin}}+\lambda A_{\lambda} S H_{u} \cdot H_{d}+f_{\mathrm{soft}}(S),
$$

with

$$
V_{\mathrm{soft}}^{\operatorname{trilin}}=-\widetilde{U}^{c} A^{U} \widetilde{Q} \cdot H_{u}+\widetilde{D}^{c} A^{D} \widetilde{Q} \cdot H_{d}+\widetilde{E}^{c} A^{L} \widetilde{L} \cdot H_{d}+\text { H.c. },
$$

where the $A$-term couplings $A_{i j}^{F}(F=U, D, L)$ are those often written as $( \pm) A_{i j}^{F} Y_{i j}^{F}$ in literature, and

$$
\begin{aligned}
V_{\mathrm{soft}}^{\mathrm{bilin}}= & \widetilde{Q}^{*} \widetilde{m}_{Q}^{2} \widetilde{Q}+\widetilde{U}^{c} \widetilde{m}_{U^{c}}^{2} \widetilde{U}^{c *}+\widetilde{D}^{c} \widetilde{m}_{D^{c}}^{2} \widetilde{D}^{c *}+\widetilde{L}^{*} \widetilde{m}_{L}^{2} \widetilde{L}+\widetilde{E}^{c} \widetilde{m}_{E^{c}}^{2} \widetilde{E}^{c *} \\
& +\widetilde{m}_{H_{u}}^{2} H_{u}^{*} H_{u}+\widetilde{m}_{H_{d}}^{2} H_{d}^{*} H_{d}+\widetilde{m}_{S}^{2} S^{*} S .
\end{aligned}
$$

The part $f_{\text {soft }}(S)$ contains the mass and self couplings of the singlet scalar. Throughout this article, the same symbol is used for the superfields $H_{u}, H_{d}, S$ and their scalar component. In all the above expression flavour indices have been suppressed. We shall return to this issue later in this section.

The gaugino mass terms:

$$
-\mathcal{L}_{\text {soft }}^{\text {gaugino }}=\frac{1}{2}\left(M_{3} \widetilde{g} \widetilde{g}+M_{2} \widetilde{W} \widetilde{W}+M_{1} \widetilde{B} \widetilde{B}\right)
$$

together with the terms in eq. (2.3) exhaust the list of soft SUSY-breaking terms in these models.

As for parts of the potential describing the singlet self interaction, in the NMSSM, $W(S)$ is usually chosen to be

$$
W(S)=\frac{1}{3} \kappa S^{3}
$$

and $V_{\text {soft }}(S)$ is therefore

$$
V_{\text {soft }}(S)=\frac{1}{3} \kappa A_{\kappa} S^{3}+\text { H.c. . }
$$

A mass term $+m^{\prime} \zeta S^{2}$ is at times added to eq. (2.7), and a corresponding one $m^{\prime} B_{\zeta} S^{2}$ in eq. (2.8) which break explicitly the scale invariance of the model.

In the nMSSM, $W(S)$ contains a linear term in $S$ :

$$
W(S)=m^{2} \xi S\left(+m^{\prime} \zeta S^{2}\right),
$$

where also an optional quadratic term in $S$, with a massive coupling $m^{\prime}$ can be added (see for example ref. [22]), and

$$
V_{\text {soft }}(S)=m^{2} \xi C_{\xi} S+\left(m^{\prime} B_{\zeta} S^{2}\right)+\text { H.c. },
$$

with $C_{\xi}$ and $B_{\zeta}$ being massive parameters. 


\begin{tabular}{|ccc|}
\hline & $\mathrm{U}(1)_{\mathrm{PQ}}$ & $\mathrm{U}(1)_{\mathrm{R}}$ \\
\hline$S$ & -2 & 2 \\
\hline$H_{u}$ & 1 & 0 \\
\hline$H_{d}$ & 1 & 0 \\
\hline$\left(U^{c} Q\right)$ & -1 & 2 \\
\hline$\left(D^{c} Q\right)$ & -1 & 2 \\
\hline$\left(E^{c} L\right)$ & -1 & 2 \\
\hline
\end{tabular}

Table 1. Peccei-Quinn and $R$-symmetry charges of the various fields.

No bilinear terms in $H_{u} \cdot H_{d}$ are present in the above superpotential, nor in the soft scalar potential terms. It is assumed in these models that such terms are generated once $S$ acquires a VEV $v_{s} \equiv\langle S\rangle$. The effective $\mu$ and $B$ parameters are then given by

$$
\begin{aligned}
& \mu_{\mathrm{eff}} \equiv \lambda v_{s}, \\
& B_{\mathrm{eff}} \equiv A_{\lambda}+\left.\frac{1}{v_{s}} \frac{\partial f^{*}\left(S^{*}\right)}{\partial S^{*}}\right|_{S=v_{s}},
\end{aligned}
$$

respectively.

Note that the mass term $m^{2}$ in eq. (2.9) may be generated spontaneously from nonrenormalizable operators of the superpotential (in the Kim-Nilles mechanism [4]) or of the Kähler potential (in the Giudice-Masiero mechanism [57]) involving the axion field, which acquires a VEV. There is in principle no particular constraint on this massive parameter $m$ to be linked with the electroweak scale. A large tadpole term, however, tend to induce a very large value of $v_{S}$. In such a case, an effective $\mu_{\text {eff }}$, compatible with electroweaksymmetry breaking, can be obtained only for a tiny value of $\lambda$ (which implies a very small singlet-doublet mixing).

As is well known, the MSSM breaks explicitly the Peccei-Quinn (PQ) symmetry through the $\mu$ term. In the MSSM with a singlet it is possible to assign PQ charges to the various fields in such a way to have $W_{\text {Yuk }}^{\mathrm{MSSM}}+\lambda S H_{u} \cdot H_{d}$ invariant under this symmetry (see first column in table 1 ).

The explicit breaking of the PQ symmetry is thus shifted into $f(S)$.

It is clear that $W_{\text {Yuk }}^{\mathrm{MSSM}}+\lambda S H_{u} \cdot H_{d}$ also enjoys an $R$ symmetry with charges for the various fields listed in the second column of table 1 . We remind that the superpotential has $R$-charge $R(W)=2$. Scalar, fermionic, and auxiliary component of the same chiral superfield $\Phi$ have different $R$-charges, with $R\left(\Phi_{\text {ferm }}\right)=R\left(\Phi_{\text {scal }}\right)-1$, and $R\left(\Phi_{\text {aux }}\right)=R\left(\Phi_{\text {scal }}\right)-2$. Moreover, the fermionic component $\lambda_{a}$ of a gauge superfield $V_{a}$, has $R$-charge $R\left(\lambda_{a}\right)=1$. Thus, the $R$ symmetry must be broken in the process of breaking SUSY, generating the $R$-violating mass terms for gauginos in eq. (2.6).

Through the VEV of $S$, induced by the electroweak-symmetry breaking, both global $\mathrm{U}(1)$ symmetries are spontaneously broken. In particular, the breaking of the PQ symmetry 
results in an axion-like CP-odd scalar component of $S$, whose mass is proportional to $\kappa$ (or $\xi$ depending on the specific model) and therefore vanishing in the limit $\kappa \rightarrow 0$ (or $\xi \rightarrow 0$ ). The limit $\kappa \rightarrow 0$ (or $\xi \rightarrow 0$ ), in contrast, does not restore the $R$ symmetry, because there are other terms in the Lagrangian that also break this symmetry.

In addition to the PQ and $R$ symmetric ones, there is also the MSSM limit, obtained for $\lambda, \kappa \rightarrow 0$ in the NMSSM (or $\lambda, \xi \rightarrow 0$ in the nMSSM) while keeping $\mu_{\text {eff }}$ finite. In this limit, $S$ tends to decouple from the model, giving rise to the superpotential and thus also to the scalar potential of the MSSM. However, the phenomenology may still be different from the one of the MSSM as the singlino (which is very weakly coupled for a finite but small $\lambda$ ) can be very light, i.e. the LSP. Therefore, expectations for DM and collider searches may differ substantially from those of the MSSM.

We close this overview of the models object of this paper with two subsections: one on the mixing of Higgs doublets and singlet, and one on the flavour basis we adopt in our investigation.

\subsection{Mixing of Higgs bosons}

The singlet Higgs boson and the neutral components of the doublet Higgs bosons mix with each other. In order to study this mixing we expand the singlet field $S$ as

$$
S=v_{s}+\frac{1}{\sqrt{2}}\left\{h_{s}+i a_{s}\right\},
$$

in analogy with $H_{u}^{0}$ and $H_{u}^{0}$ adopted in the MSSM

$$
\begin{aligned}
& H_{d}^{0}=v_{d}+\frac{1}{\sqrt{2}}\left\{h_{d}+i a_{d}\right\}, \\
& H_{u}^{0}=v_{u}+\frac{1}{\sqrt{2}}\left\{h_{u}+i a_{u}\right\},
\end{aligned}
$$

Here we have implicitly assumed that the global minimum of the Higgs scalar potential is realized by three real VEVs, $v_{u}, v_{d}$ and $v_{s}$.

The presence of interaction terms among $H_{u}^{0}, H_{d}^{0}$ and $S$ in the scalar potential, such as

$$
V_{(H-S)}=\mu_{\mathrm{eff}} \lambda^{*}\left(S+S^{*}\right)\left(v_{d} H_{d}^{0}+v_{u} H_{u}^{0}\right)-\lambda A_{\lambda} S\left(v_{u} H_{d}^{0}+v_{d} H_{u}^{0}\right)+\cdots,
$$

induces mass-mixing terms among these components. The mass terms in the basis (2.12), (2.13) are expressed as

$$
L_{\mathrm{mass}}^{\mathrm{Higgs}}=-\frac{1}{2}\left(\mathbf{h}^{T} M_{S}^{2} \mathbf{h}+\mathbf{a}^{T} M_{P}^{2} \mathbf{a}\right),
$$

with

$$
\mathbf{h}=\left(\begin{array}{c}
h_{d} \\
h_{u} \\
h_{s}
\end{array}\right), \quad \mathbf{a}=\left(\begin{array}{c}
a_{d} \\
a_{u} \\
a_{s}
\end{array}\right)
$$

The explicit expressions for the $3 \times 3$ mass matrices $M_{S}^{2}$ and $M_{P}^{2}$ in the NMSSM can be found, for example, in ref. [27, 28]. 
The CP-even (CP-odd) mass eigenstates $h_{1}, h_{2}$ and $h_{3}\left(a_{1}, a_{2}\right.$ and unphysical NambuGoldstone boson $G^{0}$ ) are given by

$$
O^{S}\left(\begin{array}{c}
h_{2} \\
h_{3} \\
h_{1}
\end{array}\right)=\left(\begin{array}{c}
h_{d} \\
h_{u} \\
h_{s}
\end{array}\right), \quad O^{P}\left(\begin{array}{c}
G^{0} \\
a_{2} \\
a_{1}
\end{array}\right)=\left(\begin{array}{c}
a_{d} \\
a_{u} \\
a_{s}
\end{array}\right),
$$

where $O^{S}$ and $O^{P}$ are $3 \times 3$ orthogonal rotation matrices, such that

$$
\begin{aligned}
\left(O^{S}\right)^{T} M_{S}^{2} O^{S} & =\operatorname{diag}\left(m_{h_{2}}^{2}, m_{h_{3}}^{2}, m_{h_{1}}^{2}\right), \\
\left(O^{P}\right)^{T} M_{P}^{2} O^{P} & =\operatorname{diag}\left(0, m_{a_{2}}^{2}, m_{a_{1}}^{2}\right) .
\end{aligned}
$$

Note that we choose our conventions in such a way that if the lightest CP-even (CP-odd) Higgs $h_{1}\left(a_{1}\right)$ is mostly singlet-like we will have small angles in the mixing matrices.

In the CP-odd Higgs sector, it is often convenient to use the "MSSM basis" as an intermediate step, to separate the Nambu-Goldstone mode $G^{0}$ from physical states. The corresponding rotation is given by

$$
\left(\begin{array}{c}
a_{d} \\
a_{u} \\
a_{s}
\end{array}\right)=O_{\beta}^{P}\left(\begin{array}{c}
G^{0} \\
A^{0} \\
a_{s}
\end{array}\right)=O_{\beta}^{P} O_{\theta_{A}}^{P}\left(\begin{array}{c}
G^{0} \\
a_{2} \\
a_{1}
\end{array}\right)
$$

with

$$
O_{\beta}^{P}=\left(\begin{array}{ccc}
\cos \beta & \sin \beta & 0 \\
-\sin \beta & \cos \beta & 0 \\
0 & 0 & 1
\end{array}\right), \quad O_{\theta_{A}}^{P}=\left(\begin{array}{ccc}
1 & 0 & 0 \\
0 & \cos \theta_{A} & \sin \theta_{A} \\
0 & -\sin \theta_{A} & \cos \theta_{A}
\end{array}\right),
$$

and $A^{0}$ is the mass eigenstate in the MSSM. With just a rewriting of the above equations, the two pseudoscalars $a_{1}$ and $a_{2}$ are then given by

$$
\begin{aligned}
& a_{1}=\sin \theta_{A} A^{0}+\cos \theta_{A} a_{s}, \\
& a_{2}=\cos \theta_{A} A^{0}-\sin \theta_{A} a_{s} .
\end{aligned}
$$

Note that our angle $\theta_{A}$ corresponds to the angle $\theta_{A}-\pi / 2$ in the existing literature. In the MSSM limit, when $\lambda \rightarrow 0$ with fixed $\mu_{\text {eff }}$, we have $\theta_{A} \rightarrow 0$. The angle $\theta_{A}$, however, can be very small also in other cases. For example, in the Peccei-Quinn limit, in which $a_{1}$ is light, it is given by (see for example [28])

$$
\sin \theta_{A}=\frac{v \sin \beta \cos \beta}{\sqrt{v_{s}^{2}+v^{2} \sin ^{2} \beta \cos ^{2} \beta}},
$$

which is greatly suppressed in the large $\tan \beta$ case.

The rotation matrix for the CP-even Higgs states involve in general three different mixing angles. In the MSSM limit, it is

$$
O^{S}=\left(\begin{array}{ccc}
-\sin \alpha & \cos \alpha & 0 \\
\cos \alpha & \sin \alpha & 0 \\
0 & 0 & 1
\end{array}\right)
$$


with $h_{2}=h$ (the SM-like Higgs) and $h_{3}=H$ (the heavy CP-even Higgs), and the angle $\alpha$ defined as in the MSSM. In this same limit, if $H, A$, and $H^{ \pm}$are assumed to be heavy and nearly degenerate, the above matrix converges to:

$$
O^{S} \rightarrow\left(\begin{array}{ccc}
\cos \beta & \sin \beta & 0 \\
\sin \beta & -\cos \beta & 0 \\
0 & 0 & 1
\end{array}\right)
$$

For reference, the physical state $H^{ \pm}$and the Goldstone mode $G^{ \pm}$of the charged Higgs bosons are the same as in the MSSM,

$$
\left(\begin{array}{l}
G^{ \pm} \\
H^{ \pm}
\end{array}\right)=\left(\begin{array}{rr}
\cos \beta & -\sin \beta \\
\sin \beta & \cos \beta
\end{array}\right)\left(\begin{array}{l}
H_{d}^{ \pm} \\
H_{u}^{ \pm}
\end{array}\right) .
$$

\subsection{Flavour parameters/violations}

We work in the super-CKM basis, in which the tree-level mass matrices, i.e. the Yukawa couplings, of the quarks and leptons in the superpotential are diagonal $[54,58,59]$. Then the Yukawa coupling matrices in the superpotential have the following forms:

$$
\left(Y_{U}\right)_{i j}=Y^{u_{i}(0)} V_{i j}^{(0)}, \quad\left(Y_{D}\right)_{i j}=Y^{d_{i}(0)} \delta_{i j}, \quad\left(Y_{L}\right)_{i j}=Y^{\ell_{i}(0)} \delta_{i j} .
$$

where $V^{(0)}$ is the bare CKM matrix, arising from the misalignment between $Y_{U}$ and $Y_{D}$, which we have accommodated in the up-quark sector but one could have equally well shifted it into the down sector. The relation of the bare Yukawa couplings $Y^{f_{i}(0)}$ and $V^{(0)}$ to the physical fermion masses will be discussed in section 4 . We also set $Y^{f_{i}(0)}(f=u, d, \ell)$ to be real and positive. The doublet superfield $Q$ is now defined as $Q=\left(V^{(0) \dagger} U, D\right)$. In this basis, the tree-level couplings of neutral Higgs bosons to quarks are, indeed, flavour diagonal. Similarly, in the trilinear SUSY-breaking terms in eq. (2.4), $A^{U}, A^{D}, A^{L}$, have to be understood as

$$
\left(A^{U}\right)_{i j}=A_{i k}^{u} V_{k j}^{(0)}, \quad\left(A^{D}\right)_{i j}=A_{i j}^{d}, \quad\left(A^{L}\right)_{i j}=A_{i j}^{\ell},
$$

where $A^{u}, A^{d}$, and $A^{\ell}$ are not necessarily diagonal and, in general, not even Hermitian.

We now turn to the flavour violation of the sfermion (squarks and slepton) sector. The squark mass matrices in the super-CKM basis $\left(\widetilde{q}_{L 1}, \widetilde{q}_{L 2}, \widetilde{q}_{L 3}, \widetilde{q}_{R 1}, \widetilde{q}_{R 2}, \widetilde{q}_{R 3}\right)$ are parametrized as

$$
\mathcal{M}_{f}^{2}=\left(\begin{array}{cc}
m_{f L L}^{2} & m_{f L R}^{2} \\
\left(m_{f L R}^{2}\right)^{\dagger} & m_{f R R}^{2}
\end{array}\right)
$$

with $m_{f X Y}^{2}(f=(u, d), X Y=(L L, R R, L R))$ being $3 \times 3$ matrices in flavour space. The submatrices of eq. (2.28) for down-type squarks are

$$
\begin{aligned}
m_{d L L}^{2} & =\widetilde{m}_{Q}^{2}, \\
m_{d R R}^{2} & =\widetilde{m}_{D^{c}}^{2} \\
\left(m_{d L R}^{2}\right)_{i j} & =-v_{d} A_{j i}^{d *}-v_{u} \mu_{\mathrm{eff}} Y^{d_{i}(0)} \delta_{i j} .
\end{aligned}
$$




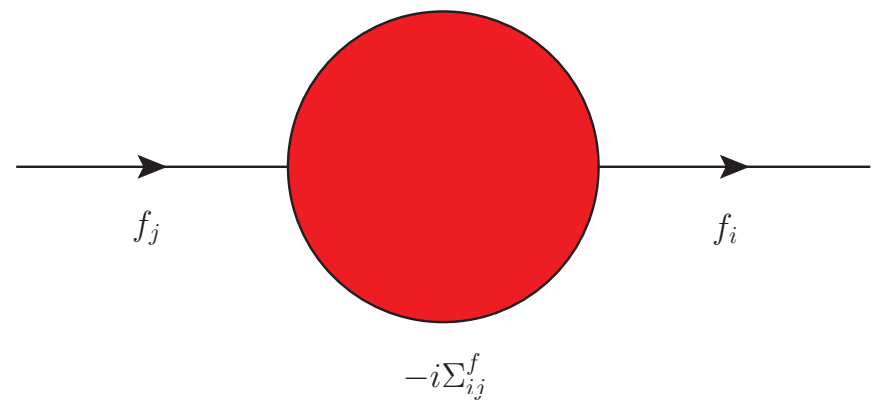

Figure 1. The diagram shows our convention for the quark and lepton self energies $\Sigma_{i j}^{f L R}$. Here $i$ and $j$ are flavour indices and $f=(u, d, \ell)$.

For up-type squarks, we have

$$
\begin{aligned}
m_{u L L}^{2} & =V^{(0)}\left(\widetilde{m}_{Q}^{2}\right) V^{(0) \dagger} \\
m_{u R R}^{2} & =\widetilde{m}_{U^{c}}^{2} \\
\left(m_{u L R}^{2}\right)_{i j} & =-v_{u} A_{j i}^{u *}-v_{d} \mu_{\mathrm{eff}} Y^{u_{i}(0)} \delta_{i j} .
\end{aligned}
$$

The mass matrix for the sleptons is obtained from $\mathcal{M}_{d}^{2}$ by replacing $\left(Q, D, A^{d}\right)$ with $\left(L, E, A^{\ell}\right)$, repspectively. We drop $O\left(v_{d, u}^{2}\right)$ terms in eqs. (2.29), (2.30) in our approximation, as explained in section 3. It should be noted that $m_{u L L}^{2}$ and $m_{d L L}^{2}$ are related by $\mathrm{SU}(2)$ invariance.

\section{Quark and lepton self-energies}

In this sections we calculate the complete set of chirality changing one-loop quark and charged-lepton self energies induced by SUSY particles (i.e. sfermions, gauginos and Higgsinos).

We decompose the self-energies of quarks and leptons $-i \Sigma_{i j}^{f}(p)$ (see figure 1) as

$$
\Sigma_{i j}^{f}(p)=\left[\Sigma_{i j}^{f L R}\left(p^{2}\right)+\not p \Sigma_{i j}^{f R R}\left(p^{2}\right)\right] P_{R}+\left[\Sigma_{i j}^{f R L}\left(p^{2}\right)+\not p \Sigma_{i j}^{f L L}\left(p^{2}\right)\right] P_{L}
$$

with incoming $(\mathrm{SM})$ fermion $f_{j}$ and outgoing fermion $f_{i}$. Here $f=(u, d, \ell)$ denotes the fermion type and $i$ and $j$ are flavour indices.

Since we know that the SUSY particles are much heavier than the SM fermions, it is possible to expand $\Sigma_{i j}^{f}(p)$ in powers of $p / m_{\mathrm{SUSY}}$. For our purpose it is sufficient to evaluate the right-handed side of eq. (3.1) at $p^{2}=0$, i.e. at leading order in $p / m_{\mathrm{SUSY}}$. Furthermore, since we are only interested in chirally-enhanced effects related to Higgs-fermion couplings we only need the chirality-changing part of the self-energies:

$$
\Sigma_{i j}^{f L R} \equiv \Sigma_{i j}^{f L R}(0)=\Sigma_{j i}^{f R L *}(0) .
$$

We further assume that the masses of the SUSY particles in the loops are sufficiently larger than the VEVs of doublet Higgs bosons, i.e. $\left(v_{d}, v_{u}\right) \ll m_{\mathrm{SUSY}}$, and evaluate $\Sigma_{i j}^{f L R}$ to leading (first) order in $v / M_{\text {SUSY }}$. We refer to this approximation as the decoupling limit 


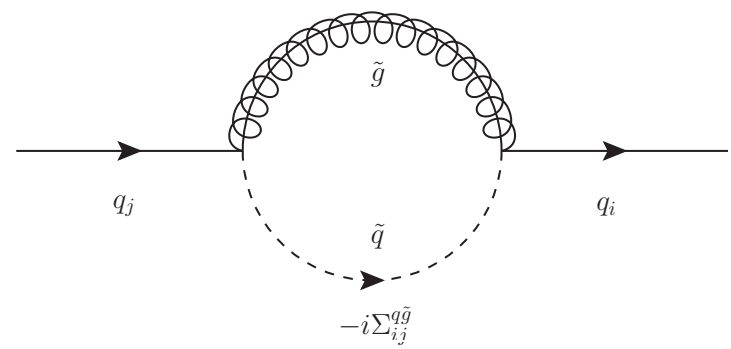

Figure 2. Quark self-energy with gluino and squark as virtual particles.

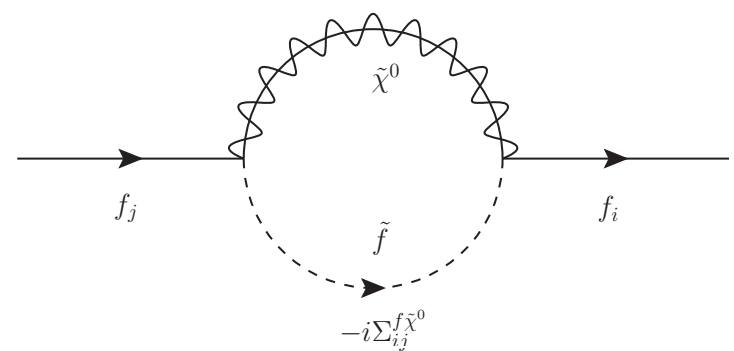

Figure 3. Fermion (quark and lepton) self-energy with sfermions and neutralinos as virtual particles.

since the remaining terms of $\Sigma_{i j}^{f L R}$ do not vanish for $\left(M_{\mathrm{SUSY}}, \mu_{\mathrm{eff}}\right) \rightarrow \infty$. For calculating $\Sigma_{i j}^{f L R}$ to leading order in $v / M_{\mathrm{SUSY}}$ the SU(2)-breaking elements of the SUSY mass matrices of the sfermions, neutralinos and charginos (such as the left-right mixing of sfermions or gaugino-higgsino mixing) are then not treated by the mixing matrices but rather by mass insertions involving $v_{d}$ or $v_{u}$. In this approach, all SUSY particles in the loops are the $\mathrm{SU}(2)_{L}$ gauge eigenstates. We also need to drop the $O\left(v_{d, u}^{2}\right)$ terms in the sfermion mass matrices, as is done in eqs. (2.29), (2.30), in order to retain the non-decoupling terms only.

The sfermion mass matrices still needs to be diagonalized due to possible flavour mixing in $m_{f L L}^{2}$ and $m_{f R R}^{2}$. Neglecting $m_{f L R}^{2}$ as explained above, the diagonalization is done as

$$
\begin{aligned}
W^{f \dagger} \mathcal{M}_{f}^{2} W^{f} & =\operatorname{diag}\left(m_{\tilde{f}_{1}^{L}}^{2}, m_{\tilde{f}_{2}^{L}}^{2}, m_{\tilde{f}_{3}^{L}}^{2}, m_{\tilde{f}_{1}^{R}}^{2}, m_{\tilde{f}_{2}^{R}}^{2}, m_{\tilde{f}_{3}^{R}}^{2}\right), \\
W^{f} & =\left(\begin{array}{cc}
W^{f L} & 0 \\
0 & W^{f R}
\end{array}\right) .
\end{aligned}
$$

The $3 \times 3$ mixing matrices $W^{f L, R}$ take into account the flavour mixing originating from the terms $m_{f L L}^{2}$ and $m_{f R R}^{2}$, respectively. Note that the relations $m_{\tilde{u}_{i}^{L}}=m_{\tilde{d}_{i}^{L}} \equiv m_{\tilde{q}_{i}^{L}}(i=1,2,3)$ and $W^{u L}=V^{(0)} W^{d L}$ are fulfilled due to $\mathrm{SU}(2)$ invariance.

For later convenience we introduce the abbreviations

$$
\begin{aligned}
\Lambda_{m i j}^{f L L} & =\left(W^{f L}\right)_{i m}\left(W^{f L \star}\right)_{j m}, \\
\Lambda_{m i j}^{f R R} & =\left(W^{f R}\right)_{i m}\left(W^{f R \star}\right)_{j m},
\end{aligned}
$$

where $i, j, m=1,2,3$. In eq. (3.4) index $m$ is not summed over.

The self energies induced by sfermion loops resemble those in the MSSM by $\mu_{\text {eff }} \leftrightarrow \mu$. Note that the elements $m_{f L R}^{2}$ in eqs. (2.29), (2.30), inserted into sfermion propagators in 


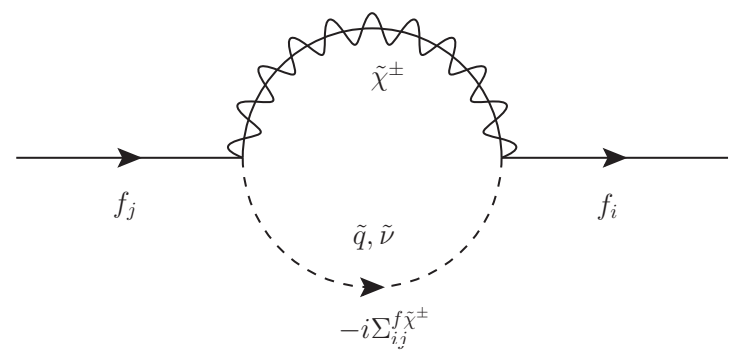

Figure 4. Fermion self-energies with sfermions and charginos as virtual particles.

the loops, may generate chirality-enhanced effects with respect to the tree-level masses if they involve the large VEV $v_{u}$ ( $\tan \beta$-enhancement for down-quark/lepton self-energies) or a trilinear $A^{f}$-term $\left(A_{i j}^{f} /\left(Y_{i j}^{f} M_{\text {SUSY }}\right)\right.$-enhancement $)$.

Below we list the relevant contribution (as calculated in ref. [54]).

We start from the gluino-squark contributions (see figure 2). In our approximation, this contribution is proportional to $m_{q L R}^{2}$ and given by

$$
\Sigma_{f i}^{d \tilde{g} L R}=\frac{2 \alpha_{s}}{3 \pi} m_{\tilde{g}} \sum_{j, k=1}^{3} \sum_{m, n=1}^{3} \Lambda_{m f j}^{d L L}\left(m_{d L R}^{2}\right)_{j k} \Lambda_{n k i}^{d R R} C_{0}\left(m_{\tilde{g}}^{2}, m_{\tilde{q}_{m}^{L}}^{2}, m_{\tilde{d}_{n}^{R}}^{2}\right) .
$$

Here $C_{0}$ is the standard three-point function $[60,61]$ at vanishing momenta:

$$
\begin{aligned}
C_{0}\left(m_{1}^{2}, m_{2}^{2}, m_{3}^{2}\right) & \equiv C_{0}\left(0,0,0 ; m_{1}^{2}, m_{2}^{2}, m_{3}^{2}\right) \\
& \equiv \frac{-i}{\pi^{2}} \int d^{4} k \frac{1}{\left[k^{2}-m_{1}^{2}+i \epsilon\right]\left[k^{2}-m_{2}^{2}+i \epsilon\right]\left[k^{2}-m_{3}^{2}+i \epsilon\right]} \\
& \equiv \frac{m_{1}^{2} m_{2}^{2} \ln \left(m_{1}^{2} / m_{2}^{2}\right)+m_{2}^{2} m_{3}^{2} \ln \left(m_{2}^{2} / m_{3}^{2}\right)+m_{3}^{2} m_{1}^{2} \ln \left(m_{3}^{2} / m_{1}^{2}\right)}{\left(m_{1}^{2}-m_{2}^{2}\right)\left(m_{2}^{2}-m_{3}^{2}\right)\left(m_{3}^{2}-m_{1}^{2}\right)} .
\end{aligned}
$$

For the neutralino-sfermion contributions to lepton and quark self-energies shown in figure 3 we get

$$
\begin{aligned}
\Sigma_{f i}^{\ell \tilde{\chi}^{0} L R}=\frac{1}{16 \pi^{2}}\{ & \sum_{j, k=1}^{3} \sum_{m, n=1}^{3} g_{1}^{2} M_{1}\left(\Lambda_{m f j}^{\ell L L}\left(m_{\ell L R}^{2}\right)_{j k} \Lambda_{n k i}^{\ell R R}\right) C_{0}\left(\left|M_{1}\right|^{2}, m_{\tilde{\ell}_{m}^{L}}^{2}, m_{\tilde{\ell}_{n}^{R}}^{2}\right) \\
& +\sum_{m=1}^{3}\left[\frac { 1 } { \sqrt { 2 } g _ { 2 } } M _ { W } \operatorname { s i n } \beta Y ^ { \ell _ { i } ( 0 ) } \Lambda _ { m f i } ^ { \ell L L } \left(g_{2}^{2} M_{2} \mu_{\mathrm{eff}} C_{0}\left(\left|M_{2}\right|^{2},\left|\mu_{\mathrm{eff}}\right|^{2}, m_{\tilde{\ell}_{m}^{L}}^{2}\right)\right.\right. \\
& \left.-g_{1}^{2} M_{1} \mu_{\mathrm{eff}} C_{0}\left(\left|M_{1}\right|^{2},\left|\mu_{\mathrm{eff}}\right|^{2}, m_{\tilde{\ell}_{m}^{L}}^{2}\right)\right) \\
& \left.\left.+g_{1}^{2} \sqrt{2} \frac{M_{W}}{g_{2}} \sin \beta M_{1} \mu_{\mathrm{eff}} Y^{\ell}(0) \Lambda_{m f i}^{\ell R R} C_{0}\left(\left|M_{1}\right|^{2},\left|\mu_{\mathrm{eff}}\right|^{2}, m_{\tilde{\ell}_{m}^{R}}^{2}\right)\right]\right\}, \quad(3.7 \\
\Sigma_{f i}^{d \tilde{\chi}^{0} L R}=\frac{1}{16 \pi^{2}}\{ & \left\{\sum_{j, k=1}^{3} \sum_{m, n=1}^{3}-\frac{1}{9} g_{1}^{2} M_{1}\left(\Lambda_{m f j}^{d L L}\left(m_{d L R}^{2}\right)_{j k} \Lambda_{n k i}^{d R R}\right) C_{0}\left(\left|M_{1}\right|^{2}, m_{\tilde{q}_{m}^{L}}^{2}, m_{\tilde{d}_{n}^{R}}^{2}\right)\right. \\
& +\sum_{m=1}^{3}\left[\frac { 1 } { \sqrt { 2 } g _ { 2 } } M _ { W } \operatorname { s i n } \beta Y ^ { d _ { i } ( 0 ) } \Lambda _ { m f i } ^ { d L L } \left(g_{2}^{2} M_{2} \mu_{\mathrm{eff}} C_{0}\left(\left|M_{2}\right|^{2},\left|\mu_{\mathrm{eff}}\right|^{2}, m_{\tilde{q}_{m}^{L}}^{2}\right)\right.\right.
\end{aligned}
$$




$$
\begin{aligned}
& \left.+\frac{g_{1}^{2}}{3} M_{1} \mu_{\mathrm{eff}} C_{0}\left(\left|M_{1}\right|^{2},\left|\mu_{\mathrm{eff}}\right|^{2}, m_{\tilde{q}_{m}^{L}}^{2}\right)\right) \\
& \left.\left.+\frac{1}{3} g_{1}^{2} \sqrt{2} \frac{M_{W}}{g_{2}} \sin \beta M_{1} \mu_{\mathrm{eff}} Y^{d_{f}(0)} \Lambda_{m f i}^{d R R} C_{0}\left(\left|M_{1}\right|^{2},\left|\mu_{\mathrm{eff}}\right|^{2}, m_{\tilde{d}_{m}^{R}}^{2}\right)\right]\right\} .
\end{aligned}
$$

Finally the chargino-sfermion contributions to lepton and down-quark self-energy (see figure 2) are given by

$$
\begin{aligned}
\Sigma_{f i}^{d \tilde{\chi}^{ \pm} L R}= & -\frac{Y^{d_{i}(0)}}{16 \pi^{2}} \mu_{\mathrm{eff}} \times \\
& \times\left[\delta_{i 3} Y^{u_{3}(0)} \sum_{m, n=1}^{3} V_{3 f}^{(0) \star} \Lambda_{m 33}^{d L L} V_{33}^{(0)}\left(m_{u L R}^{2}\right)_{33}^{*} \Lambda_{n 33}^{u R R} C_{0}\left(\left|\mu_{\mathrm{eff}}\right|^{2}, m_{\tilde{q}_{m}^{L}}^{2}, m_{\tilde{u}_{n}^{R}}^{2}\right)\right. \\
& \left.-\sqrt{2} g_{2} \sin \beta M_{W} M_{2} \sum_{m=1}^{3} \Lambda_{m f i}^{q L L} C_{0}\left(m_{\tilde{q}_{m}^{L}}^{2},\left|\mu_{\mathrm{eff}}\right|^{2},\left|M_{2}\right|^{2}\right)\right] \\
\Sigma_{f i}^{\ell \tilde{\chi}^{ \pm} L R}= & \frac{\sqrt{2} Y^{\ell_{i}(0)}}{16 \pi^{2}} \mu_{\mathrm{eff}} g_{2} \sin \beta M_{W} M_{2} \sum_{m=1}^{3} \Lambda_{m f i}^{\ell L L} C_{0}\left(m_{\tilde{\ell}_{m}^{L}}^{2},\left|\mu_{\mathrm{eff}}\right|^{2},\left|M_{2}\right|^{2}\right)
\end{aligned}
$$

In eqs. (3.8), (3.9), $O\left(v_{d}\right)$ terms of the gaugino-higgsino mixing are neglected since they do not lead to chirally-enhanced contributions and cause unnecessary complication due to their ultraviolet divergences. In addition, in the higgsino-squark-squark contribution of eq. (3.9) we have further neglected small up-type Yukawa couplings of the first two generations and multiple flavour-changes, instead of the full form

$$
\begin{aligned}
\Sigma_{f i}^{d \tilde{H}^{ \pm} L R}=-\frac{Y^{d_{i}(0)}}{16 \pi^{2}} \mu_{\mathrm{eff}} \sum_{f^{\prime}, j^{\prime}, j=1}^{3} V_{f^{\prime} f}^{(0) *} Y^{u_{f^{\prime}}(0)} & \Lambda_{n f^{\prime} j^{\prime}}^{u R R}\left(m_{u L R}^{2}\right)_{j j^{\prime}}^{*} V_{j i^{\prime}}^{(0)} \times \\
& \times \Lambda_{m i^{\prime} i}^{d L L} C_{0}\left(\left|\mu_{\mathrm{eff}}\right|^{2}, m_{\tilde{q}_{m}^{L}}^{2}, m_{\tilde{u}_{n}^{R}}^{2}\right) .
\end{aligned}
$$

By using this approximation, we can find an analytic resummation formula taking into account all chirally-enhanced corrections [54].

In contrast to down-type quarks, the up-type quark self-energies $\Sigma^{u L R}$ cannot be enhanced by $\tan \beta$. Nevertheless, an enhancement by $A_{i j}^{u} / Y_{i j}^{u} M_{\mathrm{SUSY}}$ is possible for the gluino and bino diagrams. These contributions are given as

$$
\begin{aligned}
\Sigma_{f i}^{u \tilde{g} L R} & =\frac{2 \alpha_{s}}{3 \pi} m_{\tilde{g}} \sum_{j, k, j^{\prime}, f^{\prime}=1}^{3} \sum_{m, n=1}^{3} V_{f f^{\prime}}^{(0)} \Lambda_{m f^{\prime} j^{\prime}}^{d L L} V_{j j^{\prime}}^{(0) \star}\left(m_{u L R}^{2}\right)_{j k} \Lambda_{n k i}^{u R R} C_{0}\left(m_{\tilde{g}}^{2}, m_{\tilde{q}_{m}^{L}}^{2}, m_{\tilde{u}_{n}^{R}}^{2}\right), \\
\Sigma_{f i}^{u \tilde{\chi}^{0} L R} & =\frac{1}{16 \pi^{2}} \sum_{m, n=1}^{3} \frac{2}{9} g_{1}^{2} M_{1} V_{f f^{\prime}}^{(0)} \Lambda_{m f^{\prime} j^{\prime}}^{d L L} V_{j j^{\prime}}^{(0) \star}\left(m_{u L R}^{2}\right)_{j k} \Lambda_{n k i}^{u R R} C_{0}\left(\left|M_{1}\right|^{2}, m_{\tilde{q}_{m}^{L}}^{2}, m_{\tilde{u}_{n}^{R}}^{2}\right) .
\end{aligned}
$$

We then denote the sum of all contributions as

$$
\begin{aligned}
& \Sigma_{f i}^{d L R}=\Sigma_{f i}^{d \tilde{g} L R}+\Sigma_{f i}^{d \tilde{\chi}^{0} L R}+\Sigma_{f i}^{d \tilde{\chi}^{ \pm} L R}, \\
& \Sigma_{f i}^{\ell L R}=\Sigma_{f i}^{\ell \tilde{\chi}^{0} L R}+\Sigma_{f i}^{\ell \tilde{\chi}^{ \pm} L R}, \\
& \Sigma_{f i}^{u L R}=\Sigma_{f i}^{u \tilde{g} L R}+\Sigma_{f i}^{u \tilde{\chi}^{0} L R} .
\end{aligned}
$$


As to be discussed later, the flavour off-diagonal pieces of $\Sigma_{f i}^{f L R}$ generate the flavourchanging couplings of the neutral Higgs bosons to quarks and leptons. In the case of the "minimal flavour violation" (MFV) with flavour-diagonal sfermion mass matrices, only charged higgsino contribution in eq. (3.9) cause flavour mixing originating from the CKM matrix.

\section{Renormalization and threshold corrections}

As already stated, in the fermion self-energies (3.5)-(3.9) the Yukawa couplings $Y^{f(0)}$ of fermions should be understood as the running ones of the superpotential in the singletextended SUSY SM. These couplings can be calculated from the physical masses of the fermions by properly taking into account the SUSY threshold corrections. As is well known, the chirally-enhanced corrections to the down-type quark masses may become numerically significant at large $\tan \beta[58,62-64,71-73]$ and must be resummed to all orders. In addition, the off-diagonal self-energies $\Sigma_{i j}^{f}$ cause rotation of the fermion mass eigenstates in the flavour space, which also generate difference between the bare CKM matrix $V^{(0)}$ of the superpotential and the physical one $V$.

In this section, we review the procedure of obtaining $Y^{f(0)}$ and $V^{(0)}$ from the SM running masses $m_{f_{i}}$ and CKM matrix $V_{i j}$, including the resummation of the chirally-enhanced corrections, following the results of ref. [54].

The running mass $m_{q_{i}}$ of the quark $q_{i}$ extracted from experiment using the SM prescription, is given by

$$
m_{q_{i}}=v_{q} Y^{q_{i}(0)}+\Sigma_{i i}^{q L R}, \quad(q=u, d) .
$$

Here $\Sigma_{i i}^{q L R}$ is the flavour-diagonal piece of the self-energy calculated in the previous section. Note that all terms in eq. (4.1) have to be evaluated at the same renormalization scale, i.e. the SUSY scale.

In the down-type quark sector, $\Sigma_{i i}^{d L R}$ is decomposed into the part which is proportional to a Yukawa coupling and the one which does not involve a Yukawa coupling, as

$$
\Sigma_{i i}^{d L R}=\Sigma_{i i Y_{i}}^{d L R}+\epsilon_{i}^{d} v_{u} Y^{d_{i}(0)}
$$

This decomposition is possible if we restrict ourselves to the decoupling limit where we have terms proportional to one power of $Y^{d_{i}(0)}$ at most as can been see from eq. (3.5), (3.8), and (3.9). The second term of eq. (4.2) gives chirally-enhanced corrections to the relation between the quark masses and the Yukawa couplings of the superpotential, i.e. modifies this relation via a chirally-enhanced threshold correction [58, 62-64, 71-73]. One automatically resums all chirally-enhanced corrections by inserting eq. (4.2) into eq. (4.1) and solving for $Y^{d_{i}(0) 5}$

$$
Y^{d_{i}(0)}=\frac{m_{d_{i}}-\Sigma_{i i Y_{i}}^{d L R}}{v_{d}\left(1+\tan \beta \varepsilon_{i}^{d}\right)} .
$$

\footnotetext{
${ }^{5}$ For a 2-loop analysis of the threshold corrections to the relation between the Yukawa couplings and the quark masses see ref. [65-70].
} 
The corresponding expressing for leptons follows trivially by replacing $d$ with $\ell$. In contrast, no such resummation is necessary for $Y^{u_{i}(0)}$ where only the contribution from $A$-terms can be significant:

$$
Y^{u_{i}(0)}=\frac{m_{u_{i}}-\Sigma_{i i Y_{i}}^{u L R}}{v_{u}}
$$

We now turn to the flavour-changing part of $\Sigma_{f i}^{f L R}$ which modifies the relation between the physical CKM matrix and the CKM matrix of the superpotential. In order to simplify the notation it is useful to define the quantity

$$
\sigma_{j i}^{f}=\frac{\Sigma_{j i}^{f L R}}{\max \left\{m_{f_{j}}, m_{f_{i}}\right\}},
$$

for $i \neq j$. The elements $\Sigma_{f i}^{f L R}$ contribute to the fermion mass matrices and therefore require an additional rotation with respect to the super-CKM basis to obtain the physical mass eigenstates of the fermions $\left(\psi_{i}^{f L}, \psi_{i}^{f R}\right)$

$$
\psi_{i}^{f L(R)} \rightarrow U_{i j}^{f L(R)} \psi_{j}^{f L(R)} .
$$

To leading order in small ratios of the quark masses $m_{f_{i}} / m_{f_{j}} \ll 1, U^{f L}$ then reads [74-76]

$$
U^{f L}=\left(\begin{array}{ccc}
1 & \sigma_{12}^{f} & \sigma_{13}^{f} \\
-\sigma_{12}^{f \star} & 1 & \sigma_{23}^{f} \\
-\left(\sigma_{13}^{f \star}-\sigma_{12}^{f \star} \sigma_{23}^{f \star}\right) & -\sigma_{23}^{f \star} & 1
\end{array}\right) .
$$

The corresponding expressions for $U^{f R}$ are obtained from the ones for $U^{f L}$ by the replacement $\sigma_{j i}^{f} \rightarrow \sigma_{i j}^{f \star}$.

Applying the rotations in eq. (4.7) to the $\bar{u}_{i L} d_{j L} W^{+}$vertex renormalizes the CKM matrix. The bare CKM matrix $V^{(0)}$ in eq. (2.26) can be calculated in terms of the physical CKM matrix $V$ as

$$
V^{(0)}=U^{u L} V U^{d L \dagger}
$$

However, we have to take into account that $U^{d L}$ in eq. (4.8) depends on $V^{(0)}$ through the chargino loop contribution to $\Sigma^{d L R}$. In general, an iteration procedure is necessary to calculate $V^{(0)}$ using eq. (4.8). Nevertheless, in our approximation, we find a closed form of $V^{(0)}$. We first decompose $\sigma_{f i}^{d}$ as

$$
\sigma_{f i}^{d}= \begin{cases}\widehat{\sigma}_{f 3}^{d}+\epsilon_{\mathrm{FC}}^{d} V_{3 f}^{(0) \star} V_{33}^{(0)}, & i=3 \\ \widehat{\sigma}_{f i}^{d}, & i=1,2\end{cases}
$$

so that $\widehat{\sigma}_{f i}^{d}$ does not depend on (off-diagonal) CKM elements and

$$
\varepsilon_{F C}^{d}=\frac{-1}{16 \pi^{2}} \mu_{\mathrm{eff}} \frac{Y^{d_{3}(0)}}{m_{d_{3}}} \sum_{m, n=1}^{3} Y^{u_{3}(0)} \Lambda_{m 33}^{d L L}\left(m_{u L R}^{2}\right)_{33}^{*} \Lambda_{n 33}^{u R R} C_{0}\left(\left|\mu_{\mathrm{eff}}\right|^{2}, m_{\tilde{q}_{m}^{L}}^{2}, m_{\tilde{u}_{n}^{R}}^{2}\right)
$$


In terms of the generalized Wolfenstein parametrization defined in the appendix of ref. [54], we find

$$
V^{(0)}=\left(\begin{array}{ccc}
1-\frac{\left|\widetilde{v}_{12}\right|^{2}}{2}+i \widetilde{v}_{\mathrm{Im}} & \widetilde{v}_{12} & \frac{\widetilde{v}_{13}}{1-\varepsilon_{F C}^{d}} \\
-\widetilde{v}_{12}^{\star} & 1-\frac{\left|\widetilde{v}_{12}\right|^{2}}{2}-i \widetilde{v}_{\mathrm{Im}} & \frac{\widetilde{v}_{23}}{1-\varepsilon_{F C}^{d}} \\
\frac{\widetilde{v}_{12}^{\star} \widetilde{v}_{23}^{\star}-\widetilde{v}_{13}^{\star}}{1-\varepsilon_{F C}^{d \star}} & -\frac{\widetilde{v}_{23}^{\star}}{1-\varepsilon_{F C}^{d \star}} & 1
\end{array}\right) .
$$

with

$$
\begin{aligned}
& \widetilde{v}_{12}=v_{12}+\sigma_{12}^{u}-\widehat{\sigma}_{12}^{d}, \quad \widetilde{v}_{23}=v_{23}+\sigma_{23}^{u}-\widehat{\sigma}_{23}^{d}, \\
& \widetilde{v}_{13}=v_{13}+\sigma_{13}^{u}-\widehat{\sigma}_{13}^{d}+\sigma_{12}^{u} v_{23}+\left(\widehat{\sigma}_{12}^{d}-\sigma_{12}^{u}\right) \widehat{\sigma}_{23}^{d}-v_{12} \widehat{\sigma}_{23}^{d}, \\
& \widetilde{v}_{\operatorname{Im}}=v_{12} \operatorname{Im}\left[\sigma_{12}^{u}+\widehat{\sigma}_{12}^{d}\right]-\operatorname{Im}\left[\sigma_{12}^{u} \widehat{\sigma}_{12}^{d \star}\right],
\end{aligned}
$$

and

$$
V=\left(\begin{array}{ccc}
1-\left|v_{12}\right|^{2} / 2 & v_{12} & v_{13} \\
-v_{12}^{\star} & 1-\left|v_{12}\right|^{2} / 2 & v_{23} \\
v_{12}^{\star} v_{23}^{\star}-v_{13}^{\star} & -v_{23}^{\star} & 1
\end{array}\right)
$$

We are now in a position to relate the self-energies $\Sigma_{i j}^{f L R}$ to corrections to the Higgs-fermion vertices and compute the effective Higges couplings as a function of SUSY breaking terms.

\section{$5 \quad$ Effective Higgs couplings to quarks and leptons}

In this section we compute the effective Higgs-fermion couplings in the MSSM with an additional gauge singlet superfield. For this purpose we first determine the couplings to the Higgs doublets $\left(H_{u}\right.$ and $\left.H_{d}\right)$ and the singlet $S$, and go afterwards to the physical basis with diagonal Higgs mass matrices.

\subsection{Couplings to doublet Higgs bosons}

The calculation of the effective couplings of the Higgs doublets $H_{u}$ and $H_{d}$ is the same as in the MSSM. We first decompose $\Sigma_{i j}^{f L R}$ as

$$
\begin{aligned}
\Sigma_{i j}^{(d, \ell) L R} & =E_{i j}^{(d, \ell)} v_{d}+E_{i j}^{\prime(d, \ell)} v_{u}, \\
\Sigma_{i j}^{u L R} & =E_{i j}^{u} v_{u}+E_{i j}^{\prime u} v_{d}, .
\end{aligned}
$$

The first terms on the right-handed side of eq. (5.1) is the holomorphic part, generated by the loop correction to coupling $\bar{f}_{i L} f_{j R} H_{d}^{*}$ which exists already at the tree-level and is only induced by $A$-terms in our approximation. In contrast, the second non-holomorphic term of eq. (5.1) comes from the loop-generated effective $\bar{f}_{i L} f_{j R} H_{u}$ coupling involving the effective $\mu_{\text {eff }}$ term as shown in figure 5 . The term in eq. (5.1) proportional to $v_{u}$ is always 

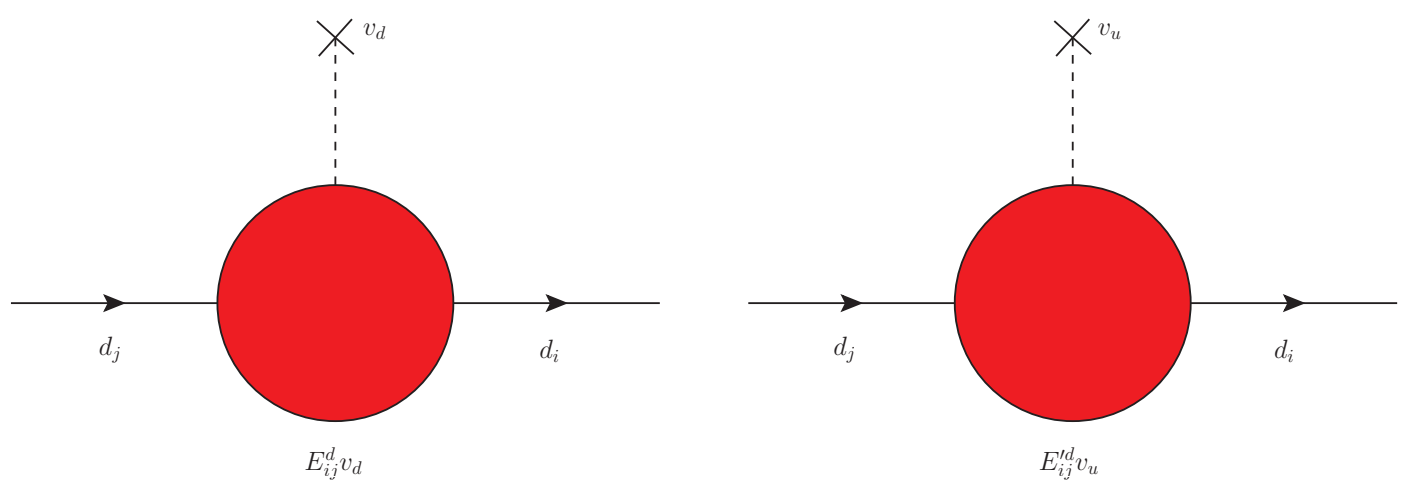

Figure 5. Decomposition of the down-type quark self-energy into its holomorphic part $E_{i j}^{d}$ and its non-holomorphic part $E_{i j}^{\prime d}$. This decomposition is possible in the decoupling limit, i.e. if $\Sigma_{i j}^{f L R}$ is evaluated at leading order in $v / m_{\mathrm{SUSY}}$. For charged leptons one simply has to replace $d$ with $\ell$.

accompanied by a factor $v_{s}$ due to the PQ symmetry. For eq. (5.2), similar discussion holds by exchanging $\left(H_{d}, v_{d}\right)$ and $\left(H_{u}, v_{u}\right)$. Note that $E_{i j}$ and $E_{i j}^{\prime}$ are general functions of $v_{s}$.

The loop contributions to the effective couplings to doublet Higgs are then obtained by replacing $v_{d}$ and $v_{u}$ in eq. (5.1) (eq. (5.2)) by $H_{d}^{*}\left(H_{d}\right)$ and $H_{u}\left(H_{u}^{*}\right)$, respectively.

\subsection{Effective singlet-fermion couplings}

We are now in a position to calculate the loop-induced couplings of the singlet Higgs $S$ to quarks and charged leptons resumming all chirally-enhanced corrections. Note that also these loop-corrections are directly related to quark and lepton self-energy generated once the singlet acquires its VEV $v_{s}$ giving rise to $\mu_{\text {eff }}$. Therefore, the effective couplings in our approach can also be calculated in terms of the chirally-enhanced quark self-energies as done before for the doublet Higgs couplings. For the couplings to the singlet we only consider down-type quarks and charged leptons since the contributions to up-type quark couplings are not chirally enhanced. We first derive the couplings in the super-CKM basis and relate them to the effective coupling in the physical basis with diagonal quark and lepton mass matrices. The effective Lagrangian governing the interactions of quarks and leptons with the singlet is given by

$$
L_{\mathrm{eff}}=\Gamma_{q_{f} q_{i}}^{h_{s} L R} h_{s} \bar{q}_{f L} q_{i R}+i \Gamma_{q_{f} q_{i}}^{a_{s} L R} a_{s} \bar{q}_{f L} q_{i R}+\text { h.c. }+(q \rightarrow \ell) .
$$

\subsubsection{Gluino and bino induced couplings}

For the effective quark-singlet coupling induced by a gluino shown in figure 6 it is sufficient to replace $\mu_{\text {eff }}$ in eq. (3.5) by $\lambda$ in order to get the corresponding effective coupling to the singlet. The same is true for the pure bino contribution to the quark or lepton self-energy. The expression for the effective gluino induced coupling then reads:

$$
\widehat{\Gamma}_{d_{f} d_{i}}^{\tilde{g} h_{s} L R}=\widehat{\Gamma}_{d_{f} d_{i}}^{\tilde{g} a_{s} L R}=-\frac{1}{\sqrt{2}} \frac{\lambda v_{u}}{\mu_{\mathrm{eff}}} E_{f i}^{\prime d \tilde{g}}
$$




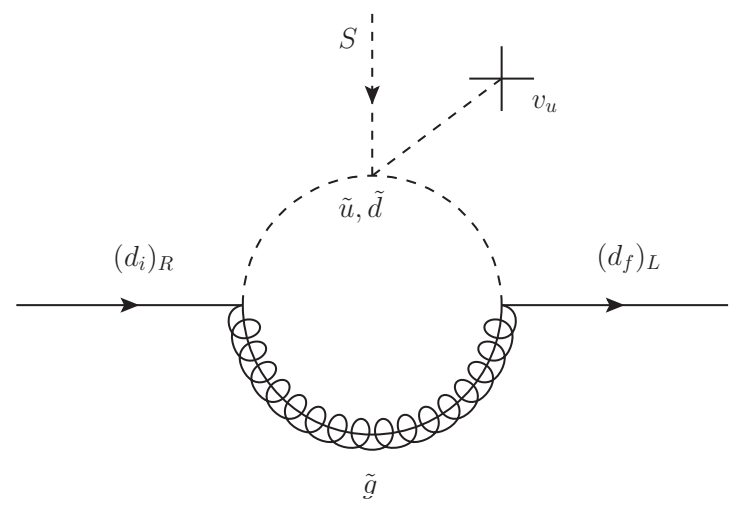

Figure 6. Feynman diagram showing the gluino contribution to the effective singlet-down-quark couplings in the decoupling limit (i.e. in leading order of $v / m_{\mathrm{SUSY}}$ ).

Here the hat refers to the fact that the couplings are given in the super-CKM basis. The expressions for the bino contribution to effective quark or lepton couplings is simply obtained by exchanging the corresponding sub- and super-scripts.

\subsubsection{Chargino and neutralino induced couplings}

Concerning the effective couplings induced by loop-diagrams with chargino and the neutralinos the situation is more involved since $\mu_{\text {eff }}$ can also appear from the diagrams like the ones shown in figure 7 . This effect is contained to all orders within the higgsino propagator emerging from the Dyson series

$$
\frac{1}{\not k}+\frac{1}{\not k} \mu_{\text {eff }} \frac{1}{\not k}+\frac{1}{\not k} \mu_{\text {eff }} \frac{1}{\not k} \mu_{\text {eff }}^{*} \frac{1}{\not k}+\frac{1}{\not k} \mu_{\text {eff }} \frac{1}{\not k} \mu_{\text {eff }}^{*} \frac{1}{\not k} \mu_{\text {eff }} \frac{1}{\not k}+\ldots=\frac{\not k+\mu_{\text {eff }}}{k^{2}-\left|\mu_{\text {eff }}^{2}\right|} .
$$

Each $\mu_{\mathrm{eff}}=\lambda v_{s}$ arises from a coupling to the singlet. In the corresponding self-energy in the decoupling limit (eq. (3.8) and eq. (3.9)) we pick out only the part of the propagator proportional to $\mu_{\mathrm{eff}}$ ( or $\mu_{\mathrm{eff}}^{*}$ ) meaning that we necessarily have an odd number of couplings to the singlet whose contributions to the Dyson series is:

$$
\frac{1}{\not k} \mu_{\mathrm{eff}} \frac{1}{\not k}+\frac{1}{\not k} \mu_{\mathrm{eff}} \frac{1}{\not k} \mu_{\mathrm{eff}}^{*} \frac{1}{\not k} \mu_{\mathrm{eff}} \frac{1}{\not k}+\ldots=\frac{\mu_{\mathrm{eff}}}{k^{2}-\left|\mu_{\mathrm{eff}}^{2}\right|} .
$$

In all except one of these couplings the singlet is replaced by its VEV $v_{s}$. If the coupling is to $S$ (and not $S^{*}$ ) the Dyson series gives

$$
\frac{1}{\not k} \lambda S \frac{1}{\not k}+2 \frac{1}{\not k} \lambda S \frac{1}{\not k} \mu_{\text {eff }}^{*} \frac{1}{\not k} \mu_{\text {eff }} \frac{1}{\not k}+\ldots=\frac{k^{2} \lambda S}{\left(k^{2}-\left|\mu_{\text {eff }}^{2}\right|\right)^{2}}=\frac{\lambda S}{k^{2}-\left|\mu_{\text {eff }}^{2}\right|}+\frac{\left|\mu_{\text {eff }}^{2}\right| \lambda S}{\left(k^{2}-\left|\mu_{\text {eff }}^{2}\right|\right)^{2}} .
$$

where the factor 2 takes into account the possible permutations. If the coupling is to $S^{*}$, on the other hand, we have instead

$$
\frac{\mu^{2} \lambda^{*} S^{*}}{\left(k^{2}-\left|\mu_{\text {eff }}^{2}\right|\right)^{2}}
$$



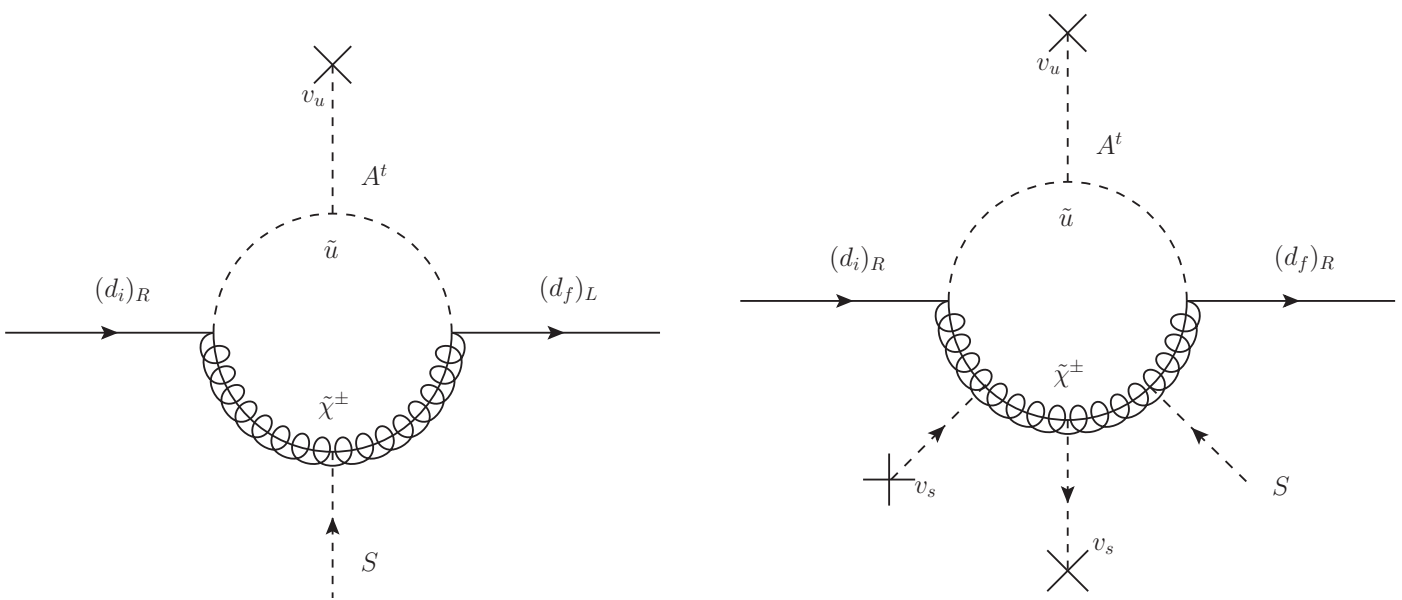

Figure 7. Example Feynman diagrams showing the chargino contribution to the effective singletdown-quark couplings in the decoupling limit (i.e. in leading order of $v / m_{\text {SUSY }}$ ).

Thus we can write for the chargino induced quark-singlet coupling in the following way:

$$
\begin{aligned}
& \widehat{\Gamma}_{d_{f} d_{i}}^{\tilde{\chi}^{ \pm} h_{s} L R}=-\frac{1}{\sqrt{2}}\left(\lambda \frac{v_{u}}{\mu_{\mathrm{eff}}} E_{f i}^{\prime d \tilde{\chi}^{ \pm}}+2 \mu_{\mathrm{eff}} \operatorname{Re}\left[\mu_{\mathrm{eff}}^{*} \lambda\right] \frac{\partial}{\partial\left|\mu_{\mathrm{eff}}^{2}\right|}\left(\frac{v_{u}}{\mu_{\mathrm{eff}}} E_{f i}^{\prime d \tilde{\chi}^{ \pm}}\right)\right), \\
& \widehat{\Gamma}_{d_{f} d_{i}}^{\tilde{\chi}^{ \pm} a_{s} L R}=-\frac{1}{\sqrt{2}}\left(\lambda \frac{v_{u}}{\mu_{\mathrm{eff}}} E_{f i}^{\prime d \tilde{\chi}^{ \pm}}+2 i \mu_{\mathrm{eff}} \operatorname{Im}\left[\mu_{\mathrm{eff}}^{*} \lambda\right] \frac{\partial}{\partial\left|\mu_{\mathrm{eff}}^{2}\right|}\left(\frac{v_{u}}{\mu_{\mathrm{eff}}} E_{f i}^{\prime d \tilde{\chi}^{ \pm}}\right)\right) .
\end{aligned}
$$

Of course also the lepton-singlet coupling induced by charginos can be written in the same way and the corresponding formula for the part of the neutralino self-energy which contains gaugino-higgsino mixing is straightforward. Note that the formulas (5.9) are also valid for gluino and bino contributions, where the second term vanishes.

We denote the sum of all contributions as:

$$
\widehat{\Gamma}_{d_{f} d_{i}}^{a_{s} L R}=\widehat{\Gamma}_{d_{f} d_{i}}^{\tilde{\chi}^{ \pm} a_{s} L R}+\widehat{\Gamma}_{d_{f} d_{i}}^{\tilde{\chi}^{0} a_{s} L R}+\widehat{\Gamma}_{d_{f} d_{i}}^{\tilde{g} a_{s} L R}, \quad \widehat{\Gamma}_{d_{f} d_{i}}^{h_{s} L R}=\widehat{\Gamma}_{d_{f} d_{i}}^{\tilde{\chi}^{ \pm} h_{s} L R}+\widehat{\Gamma}_{d_{f} d_{i}}^{\tilde{\chi}^{0} h_{s} L R}+\widehat{\Gamma}_{d_{f} d_{i}}^{\tilde{g} h_{s} L R}
$$

and for leptons $d$ is simply replaced by $\ell$.

\subsection{Higgs couplings in the physical basis}

Until now, we calculated the effective couplings of the Higgs doublets and the singlet to matter fermion. For this we worked in the interaction eigenstate for the Higgs sector and in the super-CKM basis with diagonal Yukawa couplings for the quarks and leptons. Both for quarks/leptons and for the Higgses, this is not the physical basis with diagonal mass matrices. Therefore, additional rotations (see eq. (2.17) and eq. (4.7)) are required. Note that since the masses of the quarks and leptons entirely originate from the doublets $H_{u}$ and $H_{d}$ their relation to the Yukawa couplings is the same as in the MSSM.

After integrating out the heavy SUSY particles, interactions of the doublet Higgs bosons with quarks are described by the Lagrangian

$$
\begin{aligned}
\mathcal{L}_{\text {doublet }}^{\mathrm{sCKM}}=\bar{Q}_{f L}^{a}\left[\left(Y_{i}^{d} \delta_{f i}+E_{f i}^{d}\right) \epsilon_{a b} H_{d}^{b \star}-E_{f i}^{\prime d} H_{u}^{a}\right] d_{i R} \\
\quad+\bar{Q}_{f L}^{a}\left[\left(Y_{i}^{u} \delta_{f i}+E_{f i}^{u}\right) \epsilon_{b a} H_{u}^{b \star}-E_{f i}^{\prime u} H_{d}^{a}\right] u_{i R}+\text { h.c. },
\end{aligned}
$$


with $E^{q}$ and $E^{\prime q}$ determined by eqs. (5.1) and (5.2). After EW symmetry breaking the quark mass matrices are given by:

$$
\begin{aligned}
& m_{f i}^{d}=v_{d}\left(Y_{i}^{d} \delta_{f i}+E_{f i}^{d}\right)+v_{u} E_{f i}^{\prime d}, \\
& m_{f i}^{u}=v_{u}\left(Y_{i}^{u} \delta_{f i}+E_{f i}^{u}\right)+v_{d} E_{f i}^{\prime u} .
\end{aligned}
$$

We use these relations in order to eliminate the dependence on $Y_{i}^{q}$ and $E_{f i}^{q}$ in eq. (5.11). In addition, we go to the physical basis with diagonal quark mass matrices

$$
U_{j f}^{q L \star} m_{j k}^{q} U_{k i}^{q R}=m_{q_{i}} \delta_{f i} .
$$

In the physical basis with diagonal quark mass matrices, the doublet-Higgs interactions with quarks are given by

$$
\begin{aligned}
\mathcal{L}_{\text {doublet }}= & -\bar{d}_{f L}\left[\left(\frac{m_{d_{i}}}{v_{d}} \delta_{f i}-\epsilon_{f i}^{d} \tan \beta\right) H_{d}^{0 \star}+\epsilon_{f i}^{d} H_{u}^{0}\right] d_{i R} \\
& -\bar{u}_{f L}\left[\left(\frac{m_{u_{i}}}{v_{u}} \delta_{f i}-\epsilon_{f i}^{u} \cot \beta\right) H_{u}^{0 \star}+\epsilon_{f i}^{u} H_{d}^{0}\right] u_{i R} \\
& +\bar{u}_{f L} V_{f j}\left[\frac{m_{d_{i}}}{v_{d}} \delta_{j i}-(\cot \beta+\tan \beta) \epsilon_{j i}^{d}\right] H_{d}^{+} d_{i R} \\
& +\bar{d}_{f L} V_{j f}^{\star}\left[\frac{m_{u_{i}}}{v_{u}} \delta_{j i}-(\tan \beta+\cot \beta) \epsilon_{j i}^{u}\right] H_{u}^{-} u_{i R}+\text { h.c. } .
\end{aligned}
$$

Here we defined the quantity ${ }^{6}$

$$
\epsilon_{f i}^{f} \equiv\left(U^{f L \dagger} E^{\prime f} U^{f R}\right)_{f i}
$$

which gives rise to the chirally-enhanced corrections to the Higgs couplings in the physical basis. In the Lagrangian

$$
\begin{aligned}
L_{\mathrm{eff}}=\Gamma_{q_{f} q_{i}}^{H_{k}^{0} L R} H_{k}^{0} \bar{q}_{f L} q_{i R}+i \Gamma_{q_{f} q_{i}}^{A_{k}^{0} L R} a_{k}^{0} \bar{q}_{f L} q_{i R} \\
+\Gamma_{u_{f} d_{i}}^{H^{ \pm} L R} H^{+} \bar{u}_{f L} d_{i R}+\Gamma_{d_{f} u_{i}}^{H^{ \pm}} L R H^{-} \bar{d}_{f L} u_{i R}+\text { (h.c.) },
\end{aligned}
$$

with $H_{k}^{0}=\left(h_{d}, h_{u}\right)$ and $A_{k}^{0}=\left(A^{0}, G^{0}\right)$, this leads to the following effective Higgs couplings to down-type quarks

$$
\begin{aligned}
\Gamma_{d_{f} d_{i}}^{h_{d} L R} & =-\frac{1}{\sqrt{2}}\left(\frac{m_{d_{i}}}{v_{d}} \delta_{f i}-\epsilon_{f i}^{d} \tan \beta\right), \\
\Gamma_{d_{f} d_{i}}^{h_{u} L R} & =-\frac{1}{\sqrt{2}} \epsilon_{f i}^{d}, \\
\Gamma_{d_{f} d_{i}}^{A^{0} L R} & =\frac{1}{\sqrt{2}} \sin \beta\left(\frac{m_{d_{i}}}{v_{d}} \delta_{f i}-\epsilon_{f i}^{d} \tan \beta\right),
\end{aligned}
$$

\footnotetext{
${ }^{6}$ We have dropped most of the parts contributing to $E^{\prime u}$, and therefore $\epsilon^{u}$, in eq. (3.11), since their contributions to the singlet Higgs couplings are suppressed by $\cot \beta$ and irrelevant for our study. See, for example, refs. [54, 77, 78] for the contributions of $\epsilon^{u}$ to the effective couplings of $H^{ \pm}$to up-type quarks.
} 
while the couplings of the Nambu-Goldstone boson $G^{0} \Gamma_{d_{f} d_{i}}^{G^{0} L R}=(1 / \sqrt{2}) \cos \beta\left(m_{d_{i}} / v_{d}\right) \delta_{f i}$ receive no corrections from $\epsilon^{d}$.

For leptons the neutral Higgs vertices follow trivially from the ones for downtype quarks.

The quark field rotations in eq. (5.14) also lead to a redefinition of the quark-singlet couplings from the super-CKM basis as

$$
\begin{aligned}
& \Gamma_{d_{f} d_{i}}^{h_{s}\left(a_{s}\right) L R}=U_{f^{\prime} f}^{d L *} \widehat{\Gamma}_{d_{f^{\prime}} d_{i^{\prime}}\left(a_{s}\right) L R}^{h^{\prime}} U_{i^{\prime} i}^{d R}, \\
& \Gamma_{\ell_{f} \ell_{i}}^{h_{s}\left(a_{s}\right) L R}=U_{f^{\prime} f}^{\ell L *} \widehat{\Gamma}_{\ell_{f^{\prime}} h_{i^{\prime}}}^{h_{l_{s}}\left(a_{s}\right) L R} U_{i^{\prime} i}^{\ell R} .
\end{aligned}
$$

Finally, we obtain the following couplings to the Higgs mass eigenstates:

$$
\left(\begin{array}{c}
\Gamma_{d_{f} d_{i}}^{h_{2} L R} \\
\Gamma_{d_{f} d_{i}}^{h_{3} L R} \\
\Gamma_{d_{f} d_{i}}^{h_{1} L R}
\end{array}\right)=\left(O^{S}\right)^{T}\left(\begin{array}{c}
\Gamma_{d_{f} d_{i}}^{h_{1} L R} \\
\Gamma_{d_{f} d_{i}}^{h_{u} L R} \\
\Gamma_{d_{f} d_{i}}^{h_{s} L R}
\end{array}\right), \quad\left(\begin{array}{c}
\Gamma_{d_{f} d_{i}}^{G^{0} L R} \\
\Gamma_{d_{f} d_{i}}^{a_{2} L R} \\
\Gamma_{d_{f} d_{i}}^{a_{1} L R}
\end{array}\right)=\left(O_{\theta_{A}}^{P}\right)^{T}\left(\begin{array}{c}
\Gamma_{d_{f} d_{i}}^{G^{0} L R} \\
\Gamma_{d_{f} d_{i}}^{A^{0} L R} \\
\Gamma_{d_{f} d_{i}}^{a_{s} L R}
\end{array}\right)
$$

The analogous results for charged leptons follow by replacing $d$ with $\ell$.

At this point we can already make a rough estimation of the effective singlet couplings to down-type quarks $\Gamma_{d_{f} d_{i}}^{\left(h_{s}, a_{s}\right) L R}$ (and charged leptons as well). Comparing eq. (5.9) and eq. (5.18), it is seen that the singlet couplings are suppressed by $v / v_{s}$ compared to the loop-induced couplings of the doublets $\left(h_{d}, A^{0}\right)$. As a result, the singlet couplings decouple with $\left(M_{\mathrm{SUSY}}, \mu_{\mathrm{eff}}\right) \rightarrow \infty$ and fixed $\lambda$, while the effective doublet Higgs boson couplings remain finite in the same limit. Furthermore, the scaling with $\tan \beta$ is different: while the

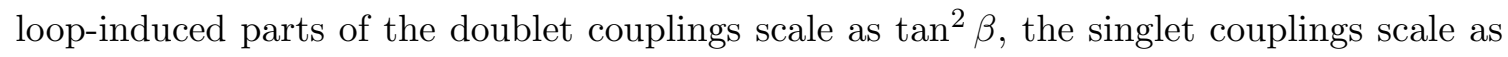
$\tan \beta$. Nevertheless, for the Higgs mass eigenstates which are almost pure singlet, as is the case for example for pseudo-axions in Peccei-Quinn symmetric limit $(2.22), \Gamma^{h_{s}, a_{s}}$ may give dominant contribution to the effective couplings to down-type quarks.

\section{$6 \quad$ Numerical results}

In this section we study the numerical significance of loop-induced effective couplings of the singlet Higgs to down-type quarks and charged leptons. We first illustrate the generic size of these couplings and then discuss the potential effect of the singlet-induced contributions to flavour physics.

In the following we will quantify the size of the effective couplings of singlet Higgs bosons to down-type quarks (charged leptons) $\Gamma_{d_{f} d_{i}}^{\left(h_{s}, a_{s}\right) L R}\left(\Gamma_{\ell_{f} \ell_{i}}^{\left(h_{s}, a_{s}\right) L R}\right)$. For the couplings to quarks we include QCD running and evaluate them at the squark mass scale. As input values for SM parameters $\left(\alpha_{s}, m_{b}, \ldots\right)$ we use the current PDG values [79]. For the SUSY sector parameters, we use the following values: $M_{1}=M_{2}=1 \mathrm{TeV}, m_{\tilde{g}}=3 \mathrm{TeV}$, $\lambda=1$. The chirality-conserving submatrix of the sfermion mass matrix $\mathcal{M}_{f}^{2}$ in eq. (2.28) is assumed to be diagonal and flavour-independent, $m_{\tilde{q}}=2 \mathrm{TeV}$ and $m_{\tilde{\ell}}=1 \mathrm{TeV}$ for squarks and sleptons, respectively. In the discussion of the flavour-changing couplings, however, we 

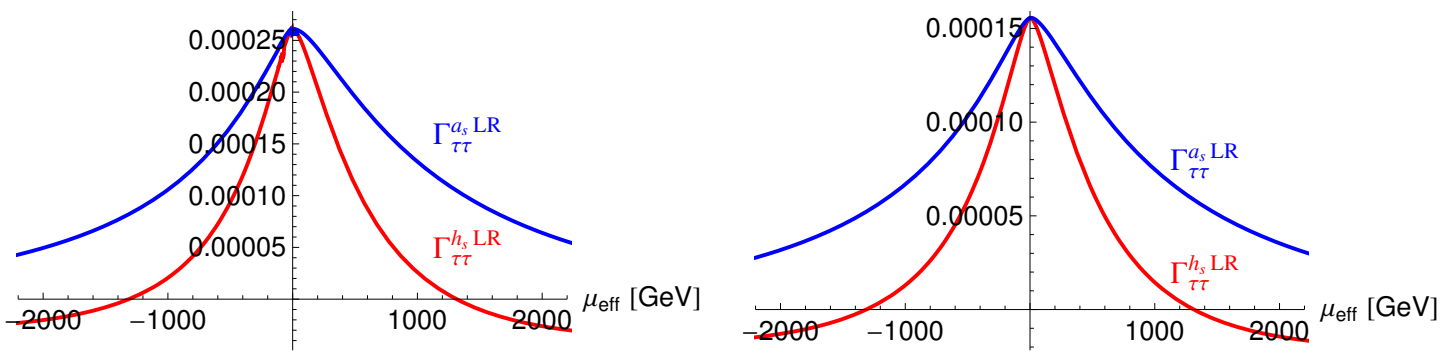

Figure 8. Left: strength of the loop-induced coupling of tau leptons to the singlet Higgs for $\tan \beta=50$ and $\lambda=1$ as a function of the (effective) $\mu$ parameter. Blue (dark gray): $h_{s}$, red (light gray): $a_{s}$. Right: same as the left plot for $\tan \beta=30$.

introduce the mixing between the third and second generations in the left-left or right-right sector. The chirality-conserving submatrix is then expressed as

$$
m_{d L L}^{2}=m_{\tilde{q}}^{2}\left(\begin{array}{ccc}
1 & 0 & 0 \\
0 & 1 & \delta_{23}^{d L L} \\
0 & \delta_{23}^{d L L} & 1
\end{array}\right),
$$

while $m_{\ell L L}^{2}$ and $m_{f R R}^{2}(f=u, d, \ell)$ are obtained by replacing $\delta_{23}^{d L L}$ by $\delta_{23}^{\ell L L}$ and $\delta_{23}^{f R R}$, respectively (and $m_{\tilde{q}} \rightarrow m_{\tilde{\ell}}$ for sleptons). The A-parameters in the chirality-changing submatrices are set to

$$
\left(A^{u}\right)_{i j}=\operatorname{diag}\left(0,0, A^{t}\right),\left(A^{d}\right)_{i j}=\operatorname{diag}\left(0,0, A^{b}\right),\left(A^{\ell}\right)_{i j}=\operatorname{diag}\left(0,0, A^{\tau}\right) .
$$

We use $A^{b}=A^{\tau}=1 \mathrm{TeV}$. Other parameters are specified in the figure captions. As stated before, we consider the case where $\mathrm{CP}$ violation is negligible and set all parameters to real.

\subsection{Flavour-conserving couplings}

We first show the flavour-conserving effective couplings of the singlet Higgs bosons to the down-type quarks and charged leptons, for the case of minimal flavour violation (MFV) $\left(\delta_{23}^{f L L}=\delta_{23}^{f R R}=0\right)$. Since these couplings are purely loop-induced, they are much smaller than the couplings to the doublet Higgs, which exists already at the tree-level. This is especially the case for charged leptons where the loop corrections cannot involve the strong interaction.

As shown in eq. (5.9), there are two types of the contributions to the singlet couplings: the one directly proportional to the non-holomorphic part of the self energy $E^{\prime f L R}$ and the other involving the derivative with respect to $\mu_{\text {eff }}$. For CP-conserving case, the latter only contribute to the couplings of the CP-even component $h_{s}$, causing the different behavior between $\Gamma^{h_{s} L R}$ and $\Gamma^{a_{s} L R}$.

Figure 8 shows the loop-induced singlet couplings to tau leptons. As expected, these couplings are much smaller than the Higgs-tau coupling in SM, $m_{\tau} /(\sqrt{2} v)=0.0072$.

For down-type quarks, the behavior of the effective couplings is similar to that for leptons, but two additional numerically important parameters enter (beside that evidently 

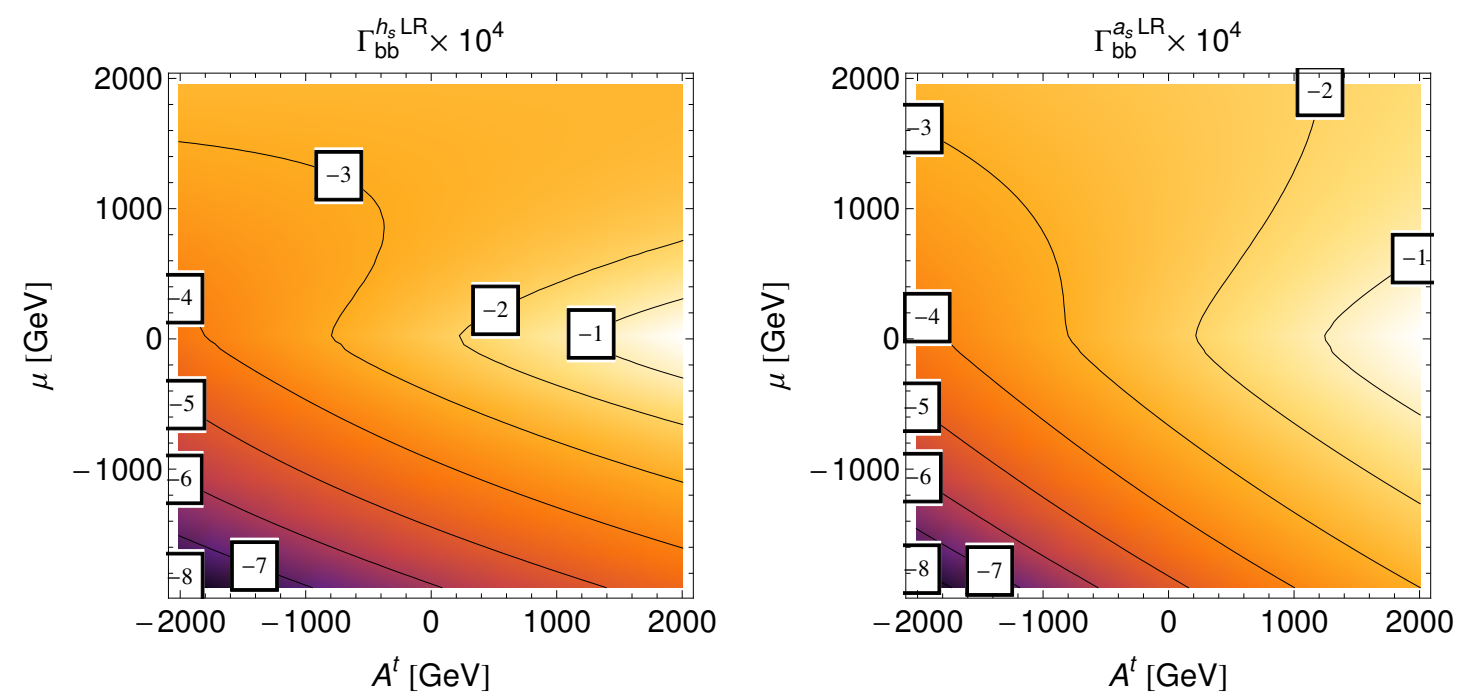

Figure 9. Left: strength of the loop-induced coupling of bottom quarks to the singlet Higgs bosons, to $h_{s}$ (left plot) and to $a_{s}$ (right plot), for $\tan \beta=50$ and $\lambda=1$ in the $\mu-A^{t}$ plane.

squark masses and slepton masses enter): $A^{t}$ and $m_{\tilde{g}}$. Furthermore, the threshold correction to the relation between $Y^{b(0)}$ and $m_{b}$ is much larger than that for $\tau$ lepton (because $\alpha_{s}$ is involved) which leads to an asymmetric behavior of $\Gamma_{b b}^{\left(h_{s}, a_{s}\right) L R}$ with respect to the sign of $\mu_{\text {eff. }}$. We show the dependence of the effective singlet coupling to bottom quarks $\Gamma_{b b}^{\left(h_{s}, a_{s}\right) L R}$ on the $\left(A^{t}, \mu_{\text {eff }}\right)$ plane in figure 9. Again, they are smaller than the coupling of the SM Higgs, $m_{b} /(\sqrt{2} v)($ at $2 \mathrm{TeV}) \sim 0.0095$.

\subsection{Flavour-changing couplings}

The flavour-changing couplings of fermions to the singlet Higgs and to the doublet Higgs are both loop-induced. As shown in section 5, the singlet couplings are suppressed by the factor $\sim\left(v / v_{s}\right) \cot \beta$ compare with the corresponding doublet couplings. Here we focus on the flavour-changing couplings between the second and third generations, induced either by the flavour mixing of left-handed sfermions $\delta_{23}^{f L L}$ or by the CKM matrices in charginosfermion loops.

\subsubsection{Leptons}

The plots of figure 10 show the behavior of effective coupling of the singlet Higgs $\Gamma_{\mu \tau}^{\left(h_{s}, a_{s}\right) L R}$ induced by $\delta_{23}^{\ell L L}$. The contribution is to a very good approximation proportional to $\delta_{23}^{\ell L L}$, unless $\delta_{23}^{\ell L L}$ is very large. The effect of $\delta_{23}^{\ell R R}$ to $\Gamma_{\mu \tau}^{\left(h_{s}, a_{s}\right) L R}$ is always suppressed by the ratio $m_{\mu} / m_{\tau}$ compared to the $\delta_{23}^{\ell L L}$ contribution, and therefore in most scenarios subleading. This is also the case for the contribution of $\delta_{23}^{\ell R R}$ to $\Gamma_{\tau \mu}^{\left(h_{s}, a_{s}\right) L R}$ since it does not involve the $\widetilde{W}$ loops.

\subsubsection{Quarks}

Let us consider first the case of the $\operatorname{MFV}\left(\delta_{23}^{q}=0\right)$. In this case only the quark-squarkchargino vertex induces flavour-violation. In figure 11 we show the size of the effective singlet couplings $\Gamma_{s b}^{\left(h_{s}, a_{s}\right) L R}$ induced via chargino loops. 

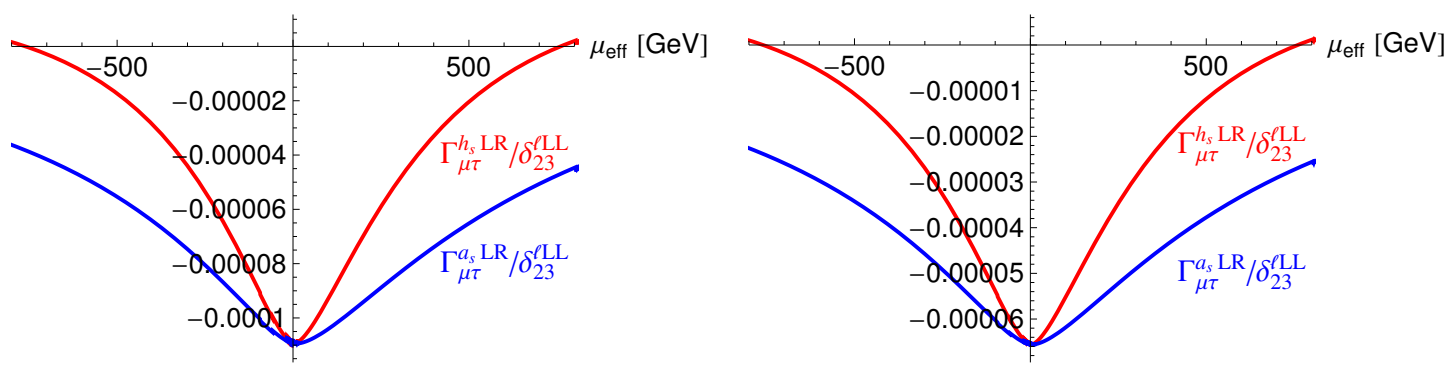

Figure 10. Left: strength of the loop-induced coupling of tau and muon to the singlet Higgs induced by $\delta_{23}^{\ell L L}$ (and normalized to $\delta_{23}^{\ell L L}$ ) for $\tan \beta=50$ and $\lambda=1$ as a function of the (effective) $\mu$ parameter. Blue (dark gray): $h_{s}$, red (light gray): $a_{s}$. Right: same as left plot for $\tan \beta=30$.
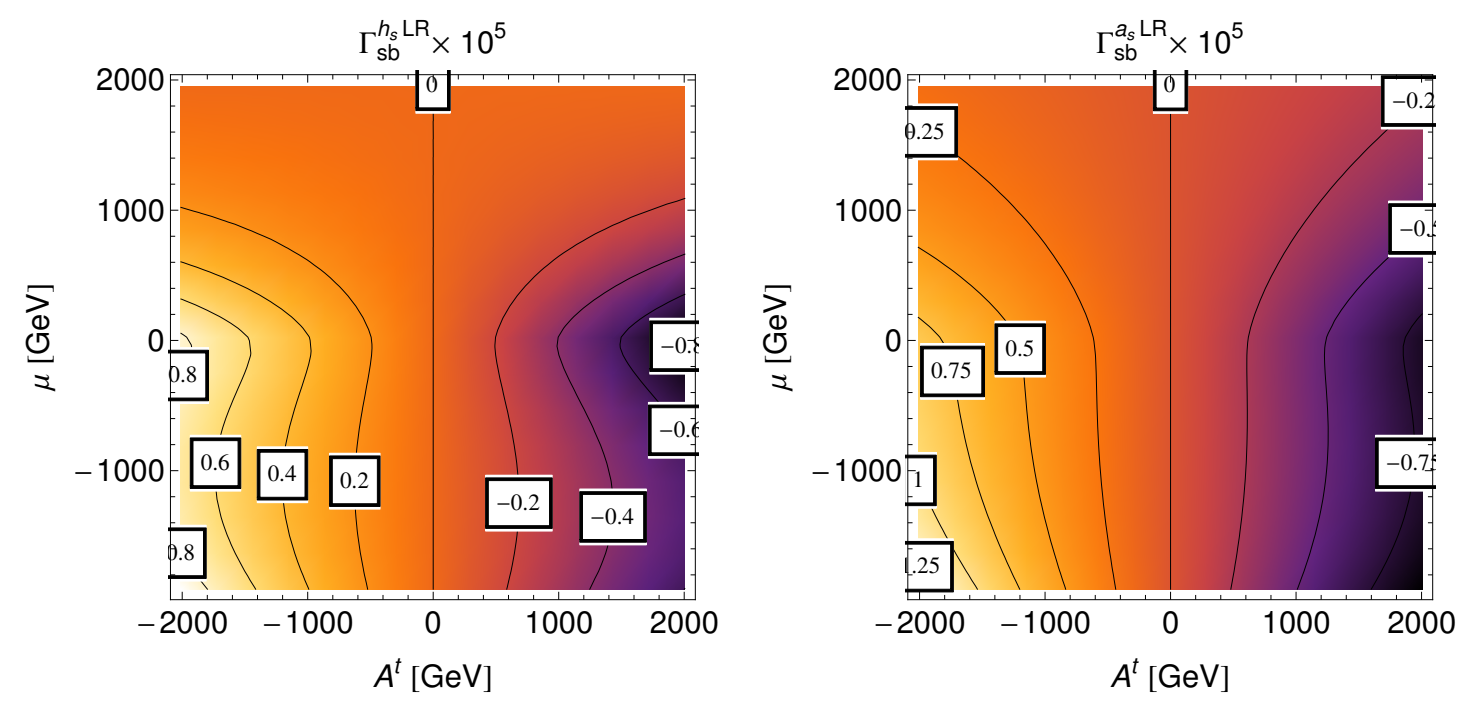

Figure 11. Size of the effective singlet couplings to bottom and strange quarks for MFV in the $\mu_{\mathrm{eff}}-A^{t}$ plane, for $\tan \beta=50$ and $\lambda=1$.

In the presence of non-minimal sources of flavour-violation in the squark sector, additional contributions are induced. In case of a non-vanishing element $\delta_{23}^{d R R}, \Gamma_{b s}^{\left(h_{s}, a_{s}\right) L R}$ is generated. Since in this case no interference with the MFV contribution occurs, their sizes are proportional to $\delta_{23}^{d R R}$. In the presence of $\delta_{23}^{d L L}$, in contrast, there is interference with the MFV contributions for $\Gamma_{s b}^{\left(h_{s}, a_{s}\right) L R}$, as seen in figure 12 .

\subsection{Effects in flavour-changing processes}

In general, the flavour-changing neutral current processes which are most sensitive to the exchange of neutral scalars which couple to quarks proportionally to their mass are $B_{s, d} \rightarrow$ $\mu^{+} \mu^{-}$and $B_{s, d}-\bar{B}_{s, d}$ mixing. For large $\tan \beta$, these processes receive potentially large contributions from the double-penguin diagrams mediated by neutral Higgs bosons [8082]. However, as discussed previously, the effective (flavour-conserving) couplings of the singlet Higgs scalars to charged leptons are much smaller than the ones of the doublet Higgs scalars. This means that the possible effect of the singlet exchange in $B_{s, d} \rightarrow \mu^{+} \mu^{-}$must be much smaller than the effect of the doublet Higgs exchange. For $B_{s, d}-\bar{B}_{s, d}$ mixing, 

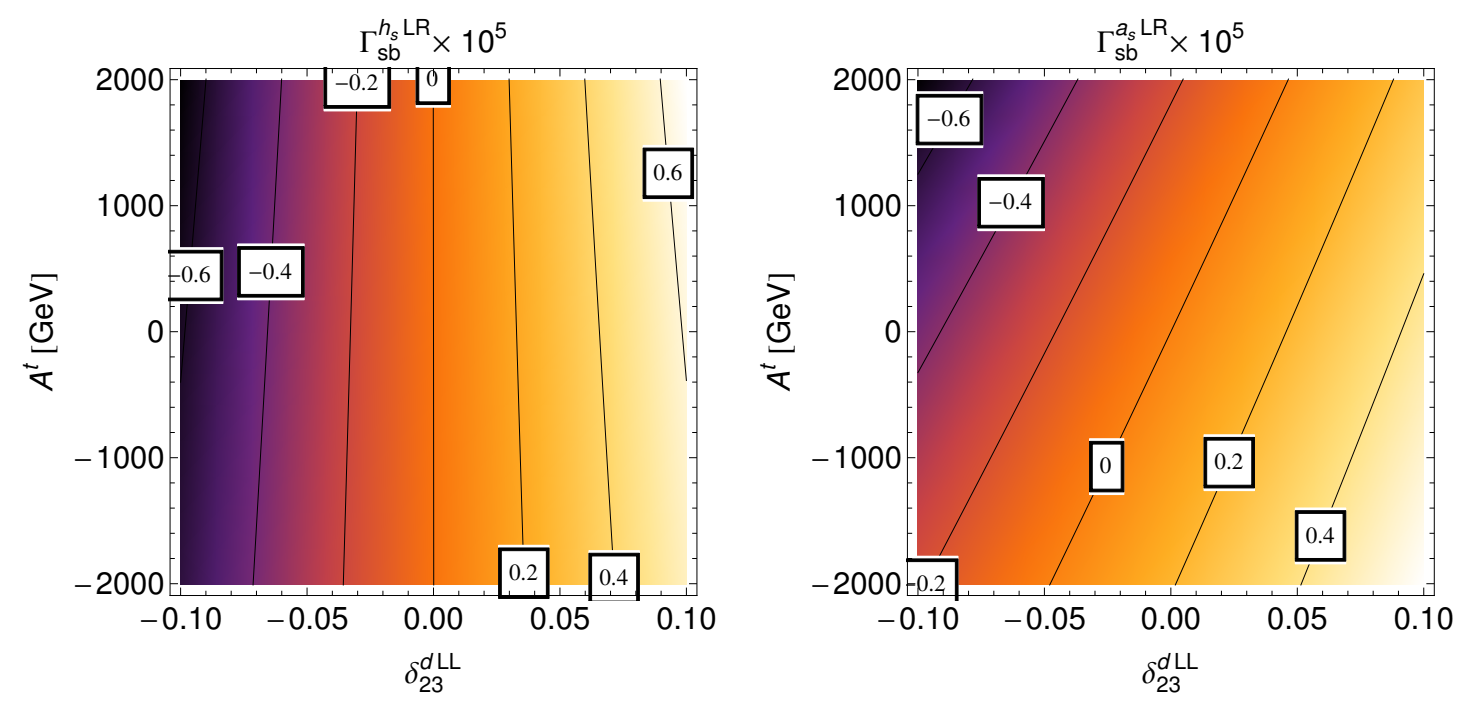

Figure 12. Size of the effective singlet couplings to bottom and strange quarks in the $A^{t}-\delta_{23}^{d L L}$ plane for $\mu=2 \mathrm{TeV}, \tan \beta=50$ and $\lambda=1$.
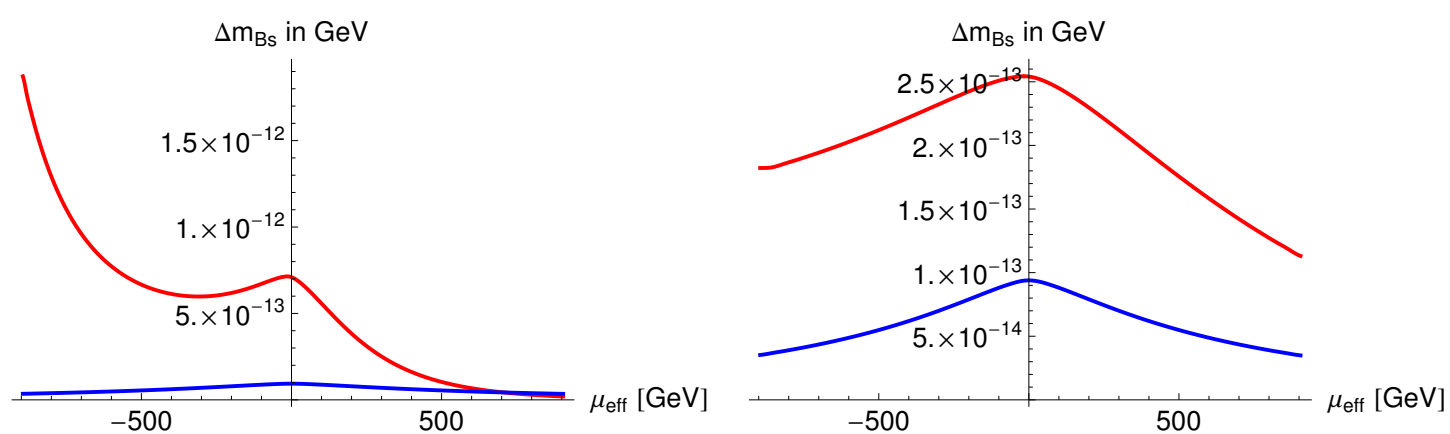

Figure 13. Contributions to the mass difference in the $B_{s}$ system generated by chargino box diagrams (blue) and by the singlet exchange (red) for $\tan \beta=50$ (left plot) and $\tan \beta=30$ (right plot) for $\lambda=1$. We assume the singlet-like $a_{s}$ has a mass of $7 \mathrm{GeV}$ while $h_{s}$ and doublet Higgs bosons are much heavier. We set $A^{t}=3 \mathrm{GeV}$.

in contrast, two loop-induced flavour-changing Higgs-quark couplings are involved in both the doublet and singlet exchanges. As a result, singlet contributions scales compared to the doublet contribution as $v^{2} m_{A}^{2} /\left(v_{s}^{2} m_{a_{s}}^{2} \tan ^{2} \beta\right)\left(m_{A}\right.$ is the typical mass of the heavier doublet Higgs bosons), which can be non-negligible for very light $a_{s}$. Furthermore, in the MFV case the doublet Higgs contribution to $B_{s, d}-\bar{B}_{s, d}$ mixing tends to chancel between the CP-even and CP-odd states having similar masses [80, 81], while such a suppression is not efficient for the singlet states where $h_{s}$ and $a_{s}$ can have very different masses.

We illustrate the relative importance of the singlet exchange in figure 13 for the MFV case and $m_{a_{s}}=7 \mathrm{GeV}$. The singlet exchange contribution is evaluated by using the effective quark-singlet couplings at the renormalization scale $m_{a_{s}}$. The meson form factors are adopted from Flavour Lattice Averaging Group (FLAG) [83]. We see that the singlet Higgs contribution can be much larger than the chargino-squark box contribution. 


\section{Conclusion}

In the framework of the MSSM extended by a gauge-singlet supermultiplet (e.g the NMSSM), we have studied the loop-induced effective couplings of the singlet Higgs bosons to quarks and charged leptons. Allowing for the most general flavour structure of the SUSY breaking terms, we have derived analytic formula for the couplings of the singlet to quarks and leptons. Keeping the leading term in the expansion of $v / M_{\text {SUSY }}$ we have performed the complete resummation of all chirally-enhanced effects, making our results valid in the phenomenologically large $\tan \beta$ region.

We found that loop-induced singlet couplings to down-type quarks and charged leptons are enhanced by one power of $\tan \beta$, while the flavour-changing couplings of down-quarks to heavy doublet Higgs bosons are enhanced by $\tan ^{2} \beta$. In addition, the loop-induced singlet-quark couplings vanish in the decoupling limit $v \ll\left(M_{\mathrm{SUSY}}, \mu_{\mathrm{eff}}\right)$ while the doublet couplings remain finite. Nevertheless, the loop-induced singlet couplings can be the dominant part of the couplings of the lightest states $a_{1}$ and/or $h_{1}$ to quarks and charged leptons if they are to a good approximation singlet like. Furthermore, these couplings can also be phenomenologically important if such $a_{1}$ is very light as it give enhanced contributions to flavour observables.

While an analysis of the impact of the effective couplings of the singlet Higgs in realistic scenarios, for example in the NMSSM, is beyond the scope of this article, we have pointed out that sizable effects of singlet Higgs in flavour observables are still possible. In our numerical analysis we have examined the generic size of the effective singlet quark (lepton) couplings, both flavour-conserving and flavour-changing ones, and considered their impact on processes where the singlet contribution is particularly relevant: for very low singlet masses, we have shown that it can be the dominant beyond-SM contribution to the $B_{s}-\bar{B}_{s}$ mixing due to the enhancement by light mass of $a_{s}$.

\section{Acknowledgments}

We are grateful to Francesca Borzumati for initializing this project and for collaboration at early stage. We thank Kwang Sik Jeong for useful discussions, and Florian Staub, Margarete Mühlleitner and Ulrich Ellwanger for informing the present status of the codes [4952]. The work of A. C. was supported by a Marie Curie Intra-European Fellowship of the European Community's 7th Framework Programme under contract number PIEF-GA2012-326948 and by the Swiss National Science Foundation.

Open Access. This article is distributed under the terms of the Creative Commons Attribution License (CC-BY 4.0), which permits any use, distribution and reproduction in any medium, provided the original author(s) and source are credited.

\section{References}

[1] P. Fayet, Supergauge invariant extension of the Higgs mechanism and a model for the electron and its neutrino, Nucl. Phys. B 90 (1975) 104 [INSPIRE]. 
[2] J.R. Ellis, J.F. Gunion, H.E. Haber, L. Roszkowski and F. Zwirner, Higgs bosons in a nonminimal supersymmetric model, Phys. Rev. D 39 (1989) 844 [InSPIRE].

[3] M. Drees, Supersymmetric models with extended Higgs sector, Int. J. Mod. Phys. A 4 (1989) 3635 [INSPIRE].

[4] J.E. Kim and H.P. Nilles, The $\mu$ problem and the strong CP problem, Phys. Lett. B 138 (1984) 150 [INSPIRE].

[5] ATLAS collaboration, Observation of a new particle in the search for the Standard Model Higgs boson with the ATLAS detector at the LHC, Phys. Lett. B 716 (2013) 1 [arXiv: 1207.7214] [INSPIRE].

[6] CMS collaboration, Observation of a new boson at a mass of $125 \mathrm{GeV}$ with the CMS experiment at the LHC, Phys. Lett. B 716 (2012) 30 [arXiv:1207.7235] [INSPIRE].

[7] J.R. Espinosa and M. Quirós, On Higgs boson masses in nonminimal supersymmetric standard models, Phys. Lett. B 279 (1992) 92 [INSPIRE].

[8] M. Masip, R. Muñoz-Tapia and A. Pomarol, Limits on the mass of the lightest Higgs in supersymmetric models, Phys. Rev. D 57 (1998) R5340 [hep-ph/9801437] [INSPIRE].

[9] J.R. Espinosa and M. Quirós, Gauge unification and the supersymmetric light Higgs mass, Phys. Rev. Lett. 81 (1998) 516 [hep-ph/9804235] [INSPIRE].

[10] M. Bastero-Gil, C. Hugonie, S.F. King, D.P. Roy and S. Vempati, Does LEP prefer the NMSSM?, Phys. Lett. B 489 (2000) 359 [hep-ph/0006198] [INSPIRE].

[11] R. Franceschini and S. Gori, Solving the $\mu$ problem with a heavy Higgs boson, JHEP 05 (2011) 084 [arXiv:1005.1070] [INSPIRE].

[12] L.J. Hall, D. Pinner and J.T. Ruderman, A natural SUSY Higgs near 126 GeV, JHEP 04 (2012) 131 [arXiv:1112.2703] [INSPIRE].

[13] U. Ellwanger and C. Hugonie, Higgs bosons near $125 \mathrm{GeV}$ in the NMSSM with constraints at the GUT scale, Adv. High Energy Phys. 2012 (2012) 625389 [arXiv:1203.5048] [INSPIRE].

[14] T. Gherghetta, B. von Harling, A.D. Medina and M.A. Schmidt, The scale-invariant NMSSM and the $126 \mathrm{GeV}$ Higgs boson, JHEP 02 (2013) 032 [arXiv:1212.5243] [INSPIRE].

[15] K.S. Jeong, Y. Shoji and M. Yamaguchi, Singlet-doublet Higgs mixing and its implications on the Higgs mass in the PQ-NMSSM, JHEP 09 (2012) 007 [arXiv:1205.2486] [INSPIRE].

[16] M. Badziak, M. Olechowski and S. Pokorski, New regions in the NMSSM with a $125 \mathrm{GeV}$ Higgs, JHEP 06 (2013) 043 [arXiv: 1304.5437] [INSPIRE].

[17] C. Panagiotakopoulos and K. Tamvakis, Stabilized NMSSM without domain walls, Phys. Lett. B 446 (1999) 224 [hep-ph/9809475] [INSPIRE].

[18] C. Panagiotakopoulos and K. Tamvakis, New minimal extension of MSSM, Phys. Lett. B 469 (1999) 145 [hep-ph/9908351] [INSPIRE].

[19] C. Panagiotakopoulos and A. Pilaftsis, Higgs scalars in the minimal nonminimal supersymmetric standard model, Phys. Rev. D 63 (2001) 055003 [hep-ph/0008268] [INSPIRE].

[20] K.S. Jeong, Y. Shoji and M. Yamaguchi, Peccei-Quinn invariant extension of the NMSSM, JHEP 04 (2012) 022 [arXiv: 1112.1014] [INSPIRE].

[21] J.E. Kim, H.P. Nilles and M.-S. Seo, Singlet superfield extension of the minimal supersymmetric standard model with Peccei-Quinn symmetry and a light pseudoscalar Higgs boson at the LHC, Mod. Phys. Lett. A 27 (2012) 1250166 [arXiv:1201.6547] [InSPIRE]. 
[22] K.J. Bae, K. Choi, E.J. Chun, S.H. Im, C.B. Park and C.S. Shin, Peccei-Quinn NMSSM in the light of $125 \mathrm{GeV}$ Higgs, JHEP 11 (2012) 118 [arXiv: 1208.2555] [INSPIRE].

[23] R.D. Peccei and H.R. Quinn, CP Conservation in the Presence of Instantons, Phys. Rev. Lett. 38 (1977) 1440 [INSPIRE].

[24] D.J. Miller, R. Nevzorov and P.M. Zerwas, The Higgs sector of the next-to-minimal supersymmetric standard model, Nucl. Phys. B 681 (2004) 3 [hep-ph/0304049] [InSPIRE].

[25] E. Accomando et al., Workshop on CP studies and non-standard Higgs physics, hep-ph/0608079.

[26] M. Artuso et al., B, D and K decays, Eur. Phys. J. C 57 (2008) 309 [arXiv:0801.1833] [INSPIRE].

[27] M. Maniatis, The next-to-minimal supersymmetric extension of the standard model reviewed, Int. J. Mod. Phys. A 25 (2010) 3505 [arXiv:0906.0777] [inSPIRE].

[28] U. Ellwanger, C. Hugonie and A.M. Teixeira, The next-to-minimal supersymmetric standard model, Phys. Rept. 496 (2010) 1 [arXiv:0910.1785] [INSPIRE].

[29] O. Lebedev and S. Ramos-Sanchez, The NMSSM and string theory, Phys. Lett. B 684 (2010) 48 [arXiv: 0912.0477] [INSPIRE].

[30] B.A. Dobrescu and K.T. Matchev, Light axion within the next-to-minimal supersymmetric standard model, JHEP 09 (2000) 031 [hep-ph/0008192] [INSPIRE].

[31] S. Chang, R. Dermisek, J.F. Gunion and N. Weiner, Nonstandard Higgs boson decays, Ann. Rev. Nucl. Part. Sci. 58 (2008) 75 [arXiv:0801.4554] [inSPIRE].

[32] A. Belyaev, J. Pivarski, A. Safonov, S. Senkin and A. Tatarinov, LHC discovery potential of the lightest NMSSM Higgs in the $h 1 \rightarrow a 1 a 1 \rightarrow 4$ muons channel, Phys. Rev. D 81 (2010) 075021 [arXiv: 1002.1956] [inSPIRE].

[33] M. Almarashi and S. Moretti, Reinforcing the no-lose theorem for NMSSM Higgs discovery at the LHC, Phys. Rev. D 84 (2011) 035009 [arXiv:1106.1599] [INSPIRE].

[34] J.-J. Cao et al., Light dark matter in NMSSM and implication on Higgs phenomenology, Phys. Lett. B 703 (2011) 292 [arXiv:1104.1754] [INSPIRE].

[35] S. Bertolini, F. Borzumati and A. Masiero, Supersymmetric contributions to $b \rightarrow s+H$, Nucl. Phys. B 312 (1989) 281 [InSPIRE].

[36] G. Hiller, B physics signals of the lightest CP odd Higgs in the NMSSM at large $\tan \beta$, Phys. Rev. D 70 (2004) 034018 [hep-ph/0404220] [INSPIRE].

[37] Z. Heng, R.J. Oakes, W. Wang, Z. Xiong and J.M. Yang, B meson dileptonic decays in the next-to-minimal supersymmetric model with a light CP-odd Higgs boson, Phys. Rev. D 77 (2008) 095012 [arXiv:0801.1169] [INSPIRE].

[38] Delphi, OPAl, ALEPH, LEP Working Group for Higgs Boson Searches, L3 collaboration, S. Schael et al., Search for neutral MSSM Higgs bosons at LEP, Eur. Phys. J. C 47 (2006) 547 [hep-ex/0602042] [INSPIRE].

[39] ALEPH collaboration, S. Schael et al., Search for neutral Higgs bosons decaying into four taus at LEP2, JHEP 05 (2010) 049 [arXiv: 1003.0705] [INSPIRE].

[40] F. Domingo, Updated constraints from radiative $\Upsilon$ decays on a light CP-odd Higgs, JHEP 04 (2011) 016 [arXiv: 1010.4701] [INSPIRE].

[41] BABAR collaboration, J.P. Lees et al., Search for hadronic decays of a light Higgs boson in the radiative decay $\Upsilon \rightarrow \gamma A^{0}$, Phys. Rev. Lett. 107 (2011) 221803 [arXiv:1108.3549] [INSPIRE]. 
[42] BABAR collaboration, J.P. Lees et al., Search for di-muon decays of a low-mass Higgs boson in radiative decays of the $\Upsilon(1 S)$, Phys. Rev. D 87 (2013) 031102 [arXiv:1210.0287] [INSPIRE].

[43] BABAR collaboration, J.P. Lees et al., Search for a light Higgs boson decaying to two gluons or $s \bar{s}$ in the radiative decays of $\Upsilon(1 S)$, Phys. Rev. D 88 (2013) 031701 [arXiv:1307.5306] [INSPIRE].

[44] CMS collaboration, Search for a light pseudoscalar Higgs boson in the dimuon decay channel in pp collisions at $\sqrt{s}=7$ TeV, Phys. Rev. Lett. 109 (2012) 121801 [arXiv:1206.6326] [INSPIRE].

[45] CMS collaboration, Search for a non-standard-model Higgs boson decaying to a pair of new light bosons in four-muon final states, Phys. Lett. B 726 (2013) 564 [arXiv:1210.7619] [INSPIRE].

[46] R.N. Hodgkinson and A. Pilaftsis, Radiative Yukawa Couplings for Supersymmetric Higgs Singlets at Large tan $\beta$, Phys. Rev. D 76 (2007) 015007 [hep-ph/0612188] [INSPIRE].

[47] R.N. Hodgkinson, $\Upsilon \rightarrow \gamma A_{1}$ in the NMSSM at large $\tan \beta$, Phys. Lett. B 665 (2008) 219 [arXiv:0802.3197] [INSPIRE].

[48] R.N. Hodgkinson and A. Pilaftsis, Supersymmetric Higgs singlet effects on B-meson FCNC observables at large tan $\beta$, Phys. Rev. D 78 (2008) 075004 [arXiv:0807.4167] [InSPIRE].

[49] J. Baglio et al., NMSSMCALC: a program package for the calculation of loop-corrected Higgs boson masses and decay widths in the (complex) NMSSM, Comput. Phys. Commun. 185 (2014) 3372 [arXiv:1312.4788] [INSPIRE].

[50] W. Porod, SPheno, a program for calculating supersymmetric spectra, SUSY particle decays and SUSY particle production at $e^{+} e^{-}$colliders, Comput. Phys. Commun. 153 (2003) 275 [hep-ph/0301101] [INSPIRE].

[51] W. Porod and F. Staub, SPheno 3.1: extensions including flavour, CP-phases and models beyond the MSSM, Comput. Phys. Commun. 183 (2012) 2458 [arXiv:1104.1573] [InSPIRE].

[52] U. Ellwanger and C. Hugonie, NMHDECAY 2.0: an updated program for sparticle masses, Higgs masses, couplings and decay widths in the NMSSM, Comput. Phys. Commun. 175 (2006) 290 [hep-ph/0508022] [INSPIRE].

[53] L. Aparicio, P.G. Camara, D.G. Cerdeno, L.E. Ibáñez and I. Valenzuela, The NMSSM with F-theory unified boundary conditions, JHEP 02 (2013) 084 [arXiv: 1212.4808] [INSPIRE].

[54] A. Crivellin, L. Hofer and J. Rosiek, Complete resummation of chirally-enhanced loop-effects in the MSSM with non-minimal sources of flavor-violation, JHEP 07 (2011) 017 [arXiv: 1103.4272] [INSPIRE].

[55] K. Cheung, T.-J. Hou, J.S. Lee and E. Senaha, The Higgs boson sector of the next-to-MSSM with CP-violation, Phys. Rev. D 82 (2010) 075007 [arXiv:1006.1458] [INSPIRE].

[56] T. Graf, R. Grober, M. Muhlleitner, H. Rzehak and K. Walz, Higgs boson masses in the complex NMSSM at one-loop level, JHEP 10 (2012) 122 [arXiv:1206.6806] [INSPIRE].

[57] G.F. Giudice and A. Masiero, A natural solution to the $\mu$ problem in supergravity theories, Phys. Lett. B 206 (1988) 480 [INSPIRE].

[58] L. Hofer, U. Nierste and D. Scherer, Resummation of tan-beta-enhanced supersymmetric loop corrections beyond the decoupling limit, JHEP 10 (2009) 081 [arXiv:0907.5408] [INSPIRE]. 
[59] A. Crivellin and U. Nierste, Chirally enhanced corrections to FCNC processes in the generic MSSM, Phys. Rev. D 81 (2010) 095007 [arXiv:0908.4404] [INSPIRE].

[60] G. 't Hooft and M.J.G. Veltman, Scalar one loop integrals, Nucl. Phys. B 153 (1979) 365 [INSPIRE].

[61] G. Passarino and M.J.G. Veltman, One loop corrections for $e^{+} e^{-}$annihilation into $\mu^{+} \mu^{-}$in the Weinberg model, Nucl. Phys. B 160 (1979) 151 [inSPIRE].

[62] T. Banks, Supersymmetry and the quark mass matrix, Nucl. Phys. B 303 (1988) 172 [INSPIRE].

[63] R. Hempfling, Yukawa coupling unification with supersymmetric threshold corrections, Phys. Rev. D 49 (1994) 6168 [INSPIRE].

[64] L.J. Hall, R. Rattazzi and U. Sarid, The top quark mass in supersymmetric $\mathrm{SO}(10)$ unification, Phys. Rev. D 50 (1994) 7048 [hep-ph/9306309] [INSPIRE].

[65] A. Bednyakov, A. Onishchenko, V. Velizhanin and O. Veretin, Two loop $O\left(\alpha_{s}^{2}\right)$ MSSM corrections to the pole masses of heavy quarks, Eur. Phys. J. C 29 (2003) 87 [hep-ph/0210258] [INSPIRE].

[66] J. Guasch, P. Hafliger and M. Spira, MSSM Higgs decays to bottom quark pairs revisited, Phys. Rev. D 68 (2003) 115001 [hep-ph/0305101] [INSPIRE].

[67] D. Noth and M. Spira, Higgs boson couplings to bottom quarks: two-loop supersymmetry-QCD corrections, Phys. Rev. Lett. 101 (2008) 181801 [arXiv:0808.0087] [INSPIRE].

[68] D. Noth and M. Spira, Supersymmetric Higgs Yukawa couplings to bottom quarks at next-to-next-to-leading order, JHEP 06 (2011) 084 [arXiv:1001.1935] [INSPIRE].

[69] A. Bauer, L. Mihaila and J. Salomon, Matching coefficients for $\alpha_{s}$ and $m(b)$ to $O\left(\alpha_{s}^{2}\right)$ in the MSSM, JHEP 02 (2009) 037 [arXiv:0810.5101] [INSPIRE].

[70] A. Crivellin and C. Greub, Two-loop supersymmetric QCD corrections to Higgs-quark-quark couplings in the generic MSSM, Phys. Rev. D 87 (2013) 015013 [Erratum ibid. D 87 (2013) 079901] [arXiv: 1210.7453] [INSPIRE].

[71] F. Borzumati, G.R. Farrar, N. Polonsky and S.D. Thomas, Soft Yukawa couplings in supersymmetric theories, Nucl. Phys. B 555 (1999) 53 [hep-ph/9902443] [INSPIRE].

[72] M. Carena, D. Garcia, U. Nierste and C.E.M. Wagner, Effective Lagrangian for the $\bar{t} b H^{+}$ interaction in the MSSM and charged Higgs phenomenology, Nucl. Phys. B 577 (2000) 88 [hep-ph/9912516] [INSPIRE].

[73] G. Isidori and A. Retico, Scalar flavor changing neutral currents in the large $\tan \beta$ limit, JHEP 11 (2001) 001 [hep-ph/0110121] [INSPIRE].

[74] A. Crivellin and U. Nierste, Supersymmetric renormalisation of the CKM matrix and new constraints on the squark mass matrices, Phys. Rev. D 79 (2009) 035018 [arXiv:0810.1613] [INSPIRE].

[75] A. Crivellin and J. Girrbach, Constraining the MSSM sfermion mass matrices with light fermion masses, Phys. Rev. D 81 (2010) 076001 [arXiv:1002.0227] [INSPIRE].

[76] A. Crivellin, Effective Higgs vertices in the generic MSSM, Phys. Rev. D 83 (2011) 056001 [arXiv: 1012.4840] [INSPIRE].

[77] G. Degrassi, P. Gambino and G.F. Giudice, $B \rightarrow X(s \gamma)$ in supersymmetry: large contributions beyond the leading order, JHEP 12 (2000) 009 [hep-ph/0009337] [INSPIRE]. 
[78] M. Carena, D. Garcia, U. Nierste and C.E.M. Wagner, $b \rightarrow s \gamma$ and supersymmetry with large $\tan \beta$, Phys. Lett. B 499 (2001) 141 [hep-ph/0010003] [INSPIRE].

[79] Particle Data Group collaboration, K.A. Olive et al., Review of particle physics, Chin. Phys. C 38 (2014) 090001 [inSPIRE].

[80] K.S. Babu and C.F. Kolda, Higgs mediated $B^{0} \rightarrow \mu^{+} \mu^{-}$in minimal supersymmetry, Phys. Rev. Lett. 84 (2000) 228 [hep-ph/9909476] [INSPIRE].

[81] A.J. Buras, P.H. Chankowski, J. Rosiek and L. Slawianowska, $\Delta M_{d, s}, B^{0} d, s \rightarrow \mu^{+} \mu^{-}$and $B \rightarrow X_{s} \gamma$ in supersymmetry at large $\tan \beta$, Nucl. Phys. B 659 (2003) 3 [hep-ph/0210145] [INSPIRE].

[82] F. Domingo and U. Ellwanger, Updated constraints from B physics on the MSSM and the NMSSM, JHEP 12 (2007) 090 [arXiv:0710.3714] [INSPIRE].

[83] S. Aoki et al., Review of lattice results concerning low-energy particle physics, Eur. Phys. J. C 74 (2014) 2890 [arXiv:1310.8555] [INSPIRE]. 